Network Working Group

Request for Comments: 3489

Category: Standards Track
J. Rosenberg

J. Weinberger dynamicsoft C. Huitema Microsoft R. Mahy Cisco March 2003

\title{
STUN - Simple Traversal of User Datagram Protocol (UDP) Through Network Address Translators (NATs)
}

Status of this Memo

This document specifies an Internet standards track protocol for the Internet community, and requests discussion and suggestions for improvements. Please refer to the current edition of the "Internet Official Protocol Standards" (STD 1) for the standardization state and status of this protocol. Distribution of this memo is unlimited.

Copyright Notice

Copyright (C) The Internet Society (2003). All Rights Reserved.

Abstract

Simple Traversal of User Datagram Protocol (UDP) Through Network Address Translators (NATS) (STUN) is a lightweight protocol that allows applications to discover the presence and types of NATs and firewalls between them and the public Internet. It also provides the ability for applications to determine the public Internet Protocol (IP) addresses allocated to them by the NAT. STUN works with many existing NATs, and does not require any special behavior from them. As a result, it allows a wide variety of applications to work through existing NAT infrastructure.

Table of Contents

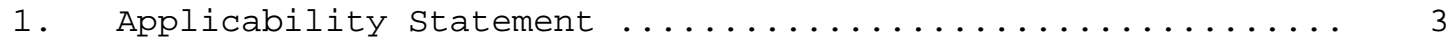

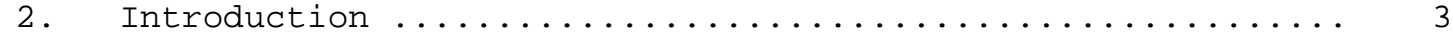

3. Terminology $\ldots \ldots \ldots \ldots \ldots \ldots \ldots \ldots \ldots \ldots \ldots \ldots \ldots \ldots \ldots \ldots \ldots$

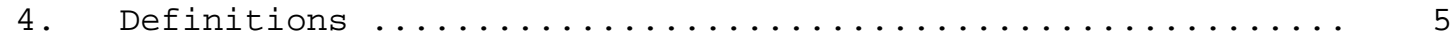

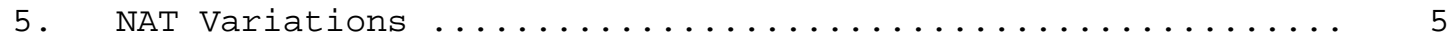

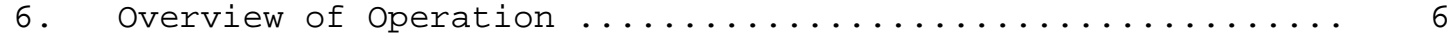

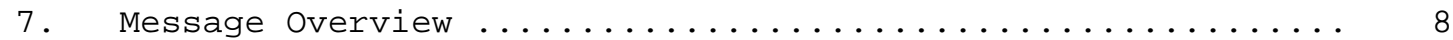

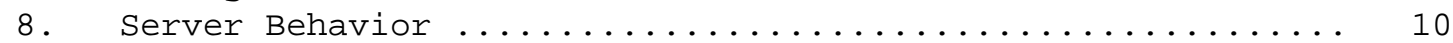

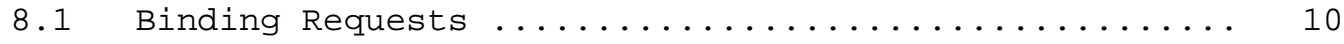




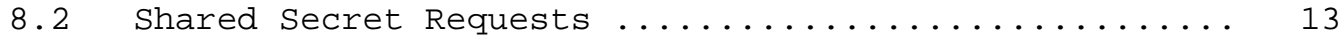

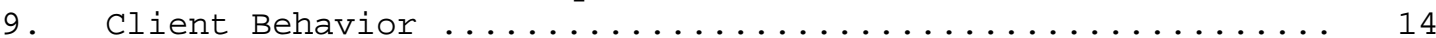

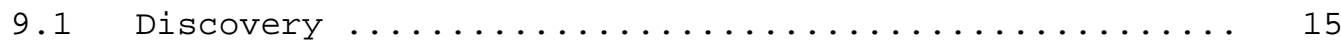

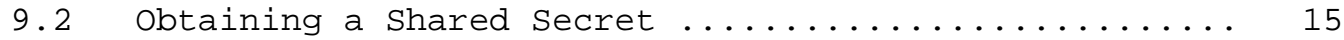

9.3 Formulating the Binding Request .............. 17

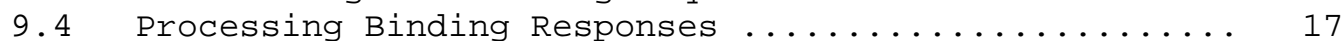

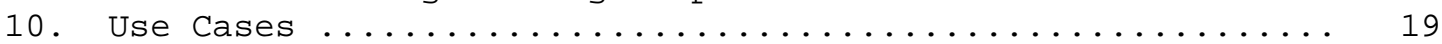

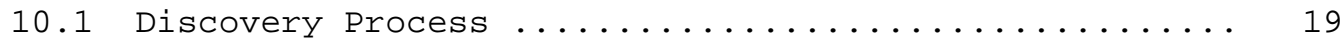

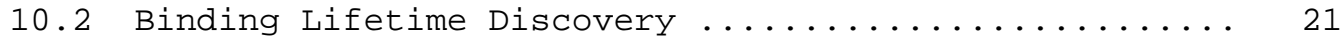

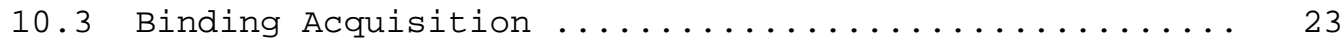

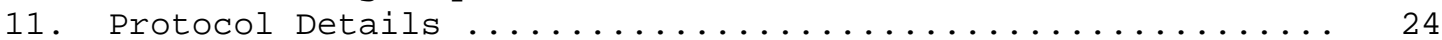

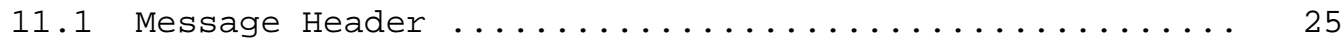

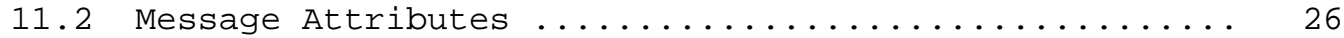

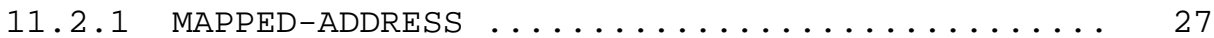

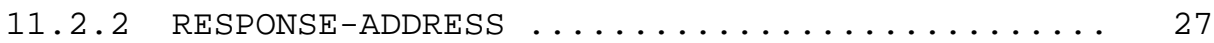

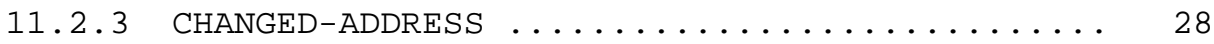

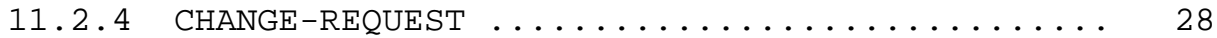

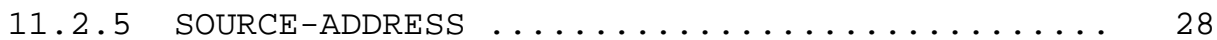

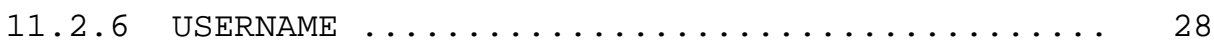

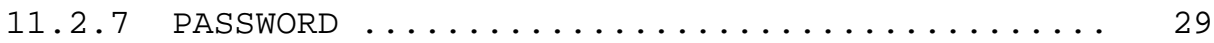

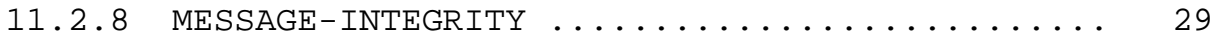

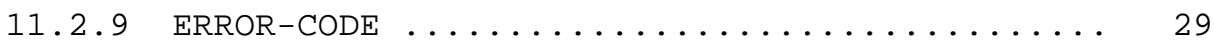

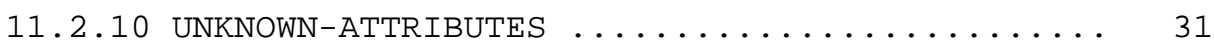

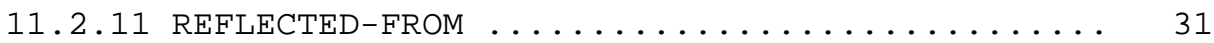

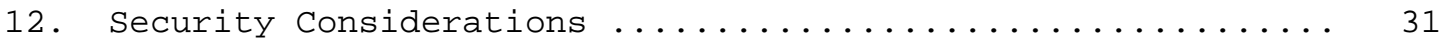

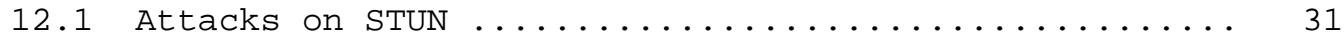

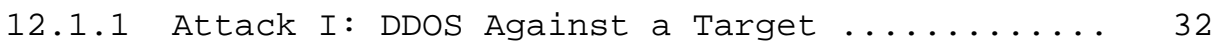

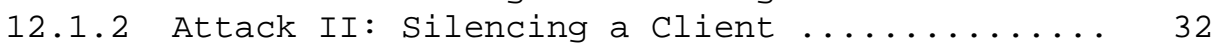

12.1.3 Attack III: Assuming the Identity of a Client 32

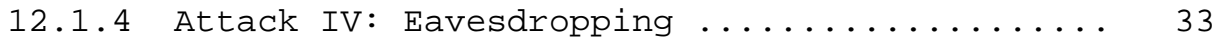

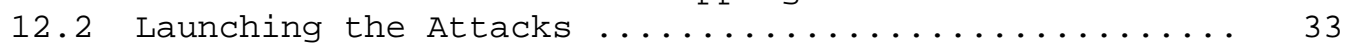

12.2.1 Approach I: Compromise a Legitimate

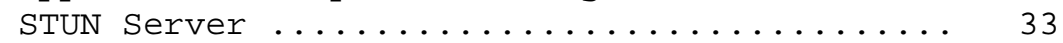

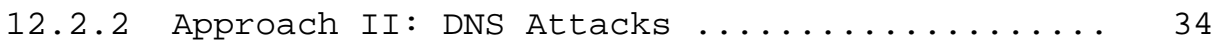

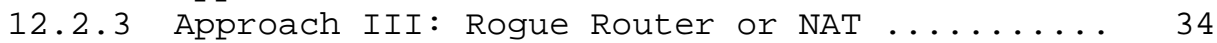

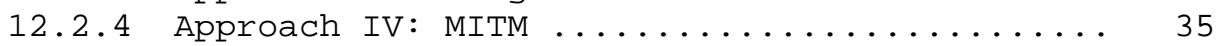

12.2.5 Approach V: Response Injection Plus DoS .... 35

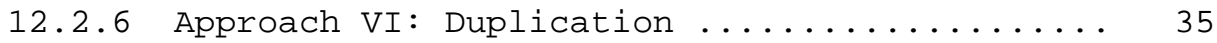

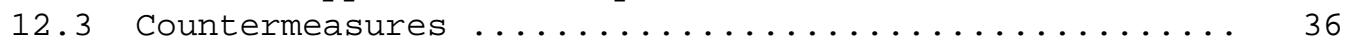

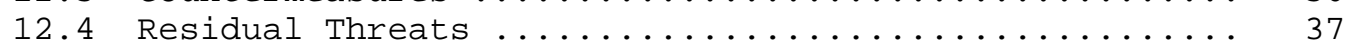

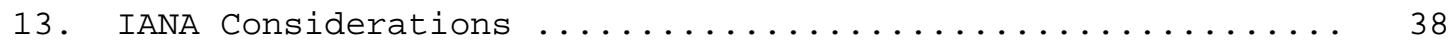

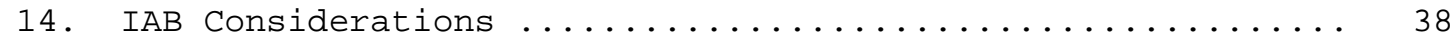

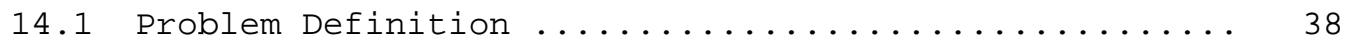

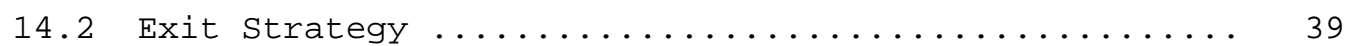

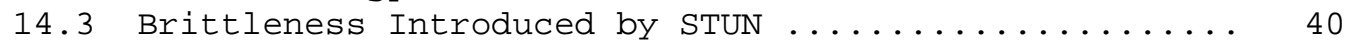

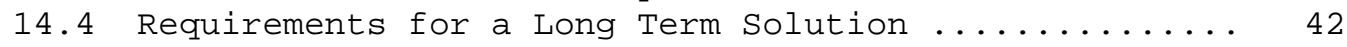

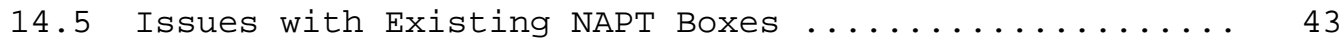

14.6 In Closing ........................... 43

Rosenberg, et al. Standards Track [Page 2] 
15. Acknowledgments ........................... 44

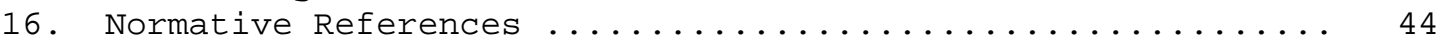

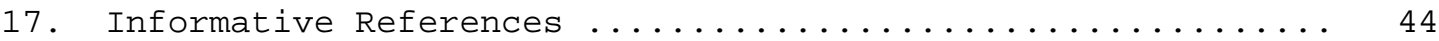

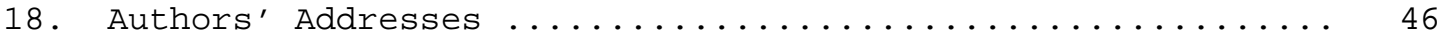

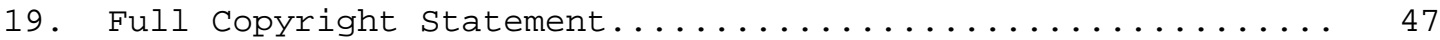

1. Applicability statement

This protocol is not a cure-all for the problems associated with NAT. It does not enable incoming TCP connections through NAT. It allows incoming UDP packets through NAT, but only through a subset of existing NAT types. In particular, STUN does not enable incoming UDP packets through symmetric NATs (defined below), which are common in large enterprises. STUN's discovery procedures are based on assumptions on NAT treatment of UDP; such assumptions may prove invalid down the road as new NAT devices are deployed. STUN does not work when it is used to obtain an address to communicate with a peer which happens to be behind the same NAT. STUN does not work when the STUN server is not in a common shared address realm. For a more complete discussion of the limitations of STUN, see section 14.

\section{Introduction}

Network Address Translators (NATs), while providing many benefits, also come with many drawbacks. The most troublesome of those drawbacks is the fact that they break many existing IP applications, and make it difficult to deploy new ones. Guidelines have been developed [8] that describe how to build "NAT friendly" protocols, but many protocols simply cannot be constructed according to those guidelines. Examples of such protocols include almost all peer-topeer protocols, such as multimedia communications, file sharing and games.

To combat this problem, Application Layer Gateways (ALGs) have been embedded in NATs. ALGs perform the application layer functions required for a particular protocol to traverse a NAT. Typically, this involves rewriting application layer messages to contain translated addresses, rather than the ones inserted by the sender of the message. ALGs have serious limitations, including scalability, reliability, and speed of deploying new applications. To resolve these problems, the Middlebox Communications (MIDCOM) protocol is being developed [9]. MIDCOM allows an application entity, such as an end client or network server of some sort (like a session Initiation Protocol (SIP) proxy [10]) to control a NAT (or firewall), in order to obtain NAT bindings and open or close pinholes. In this way, NATs and applications can be separated once more, eliminating the need for embedding ALGs in NATs, and resolving the limitations imposed by current architectures. 
Unfortunately, MIDCOM requires upgrades to existing NAT and firewalls, in addition to application components. Complete upgrades of these NAT and firewall products will take a long time, potentially years. This is due, in part, to the fact that the deployers of NAT and firewalls are not the same people who are deploying and using applications. As a result, the incentive to upgrade these devices will be low in many cases. Consider, for example, an airport Internet lounge that provides access with a NAT. A user connecting to the NATed network may wish to use a peer-to-peer service, but cannot, because the NAT doesn't support it. Since the administrators of the lounge are not the ones providing the service, they are not motivated to upgrade their NAT equipment to support it, using either an ALG, or MIDCOM.

Another problem is that the MIDCOM protocol requires that the agent controlling the middleboxes know the identity of those middleboxes, and have a relationship with them which permits control. In many configurations, this will not be possible. For example, many cable access providers use NAT in front of their entire access network. This NAT could be in addition to a residential NAT purchased and operated by the end user. The end user will probably not have a control relationship with the NAT in the cable access network, and may not even know of its existence.

Many existing proprietary protocols, such as those for online games (such as the games described in RFC 3027 [11]) and Voice over IP, have developed tricks that allow them to operate through NATs without changing those NATs. This document is an attempt to take some of those ideas, and codify them into an interoperable protocol that can meet the needs of many applications.

The protocol described here, Simple Traversal of UDP Through NAT (STUN), allows entities behind a NAT to first discover the presence of a NAT and the type of NAT, and then to learn the addresses bindings allocated by the NAT. STUN requires no changes to NATs, and works with an arbitrary number of NATs in tandem between the application entity and the public Internet.

3. Terminology

In this document, the key words "MUST", "MUST NOT", "REQUIRED", "SHALL", "SHALL NOT", "SHOULD", "SHOULD NOT", "RECOMMENDED", "MAY", and "OPTIONAL" are to be interpreted as described in BCP 14, RFC 2119 [1] and indicate requirement levels for compliant STUN implementations. 


\section{Definitions}

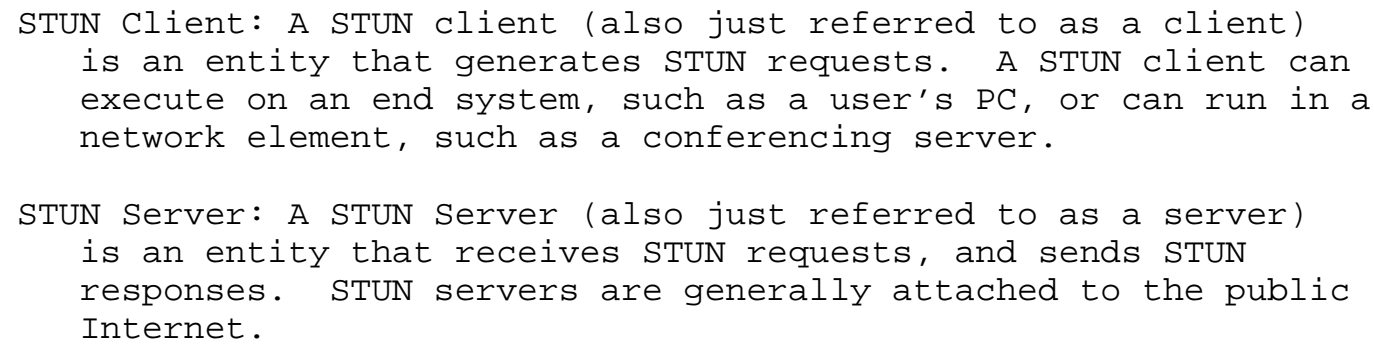

5. NAT Variations

It is assumed that the reader is familiar with NATs. It has been observed that NAT treatment of UDP varies among implementations. The four treatments observed in implementations are:

Full Cone: A full cone NAT is one where all requests from the same internal IP address and port are mapped to the same external IP address and port. Furthermore, any external host can send a packet to the internal host, by sending a packet to the mapped external address.

Restricted Cone: A restricted cone NAT is one where all requests from the same internal IP address and port are mapped to the same external IP address and port. Unlike a full cone NAT, an external host (with IP address X) can send a packet to the internal host only if the internal host had previously sent a packet to IP address $\mathrm{X}$.

Port Restricted Cone: A port restricted cone NAT is like a restricted cone $\mathrm{NAT}$, but the restriction includes port numbers. Specifically, an external host can send a packet, with source IP address $X$ and source port $P$, to the internal host only if the internal host had previously sent a packet to IP address $X$ and port $\mathrm{P}$.

Symmetric: A symmetric NAT is one where all requests from the same internal IP address and port, to a specific destination IP address and port, are mapped to the same external IP address and port. If the same host sends a packet with the same source address and port, but to a different destination, a different mapping is used. Furthermore, only the external host that receives a packet can send a UDP packet back to the internal host. 
Determining the type of NAT is important in many cases. Depending on what the application wants to do, it may need to take the particular behavior into account.

\section{Overview of Operation}

This section is descriptive only. Normative behavior is described in Sections 8 and 9 .
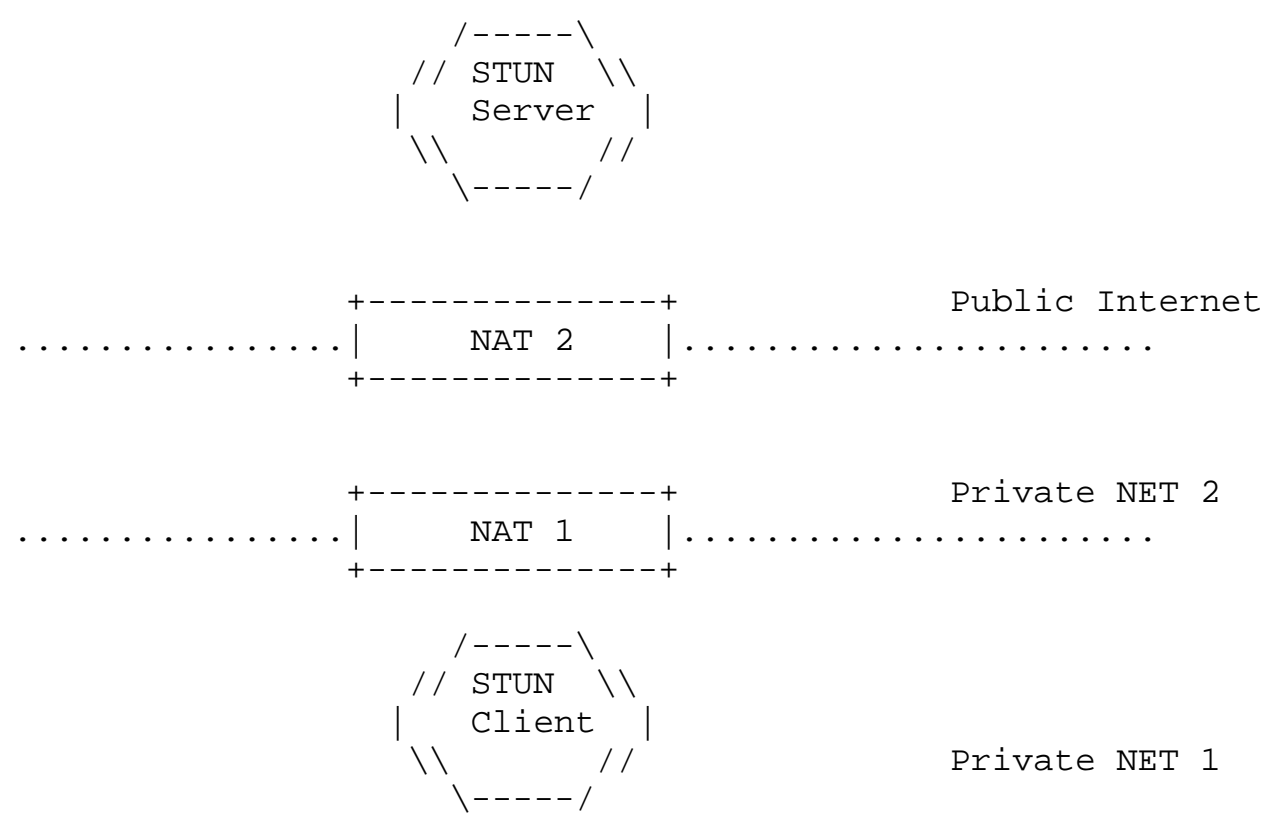

Figure 1: STUN Configuration

The typical STUN configuration is shown in Figure 1. A STUN client is connected to private network 1. This network connects to private network 2 through NAT 1. Private network 2 connects to the public Internet through NAT 2. The STUN server resides on the public Internet.

STUN is a simple client-server protocol. A client sends a request to a server, and the server returns a response. There are two types of requests - Binding Requests, sent over UDP, and Shared Secret Requests, sent over TLS [2] over TCP. Shared Secret Requests ask the server to return a temporary username and password. This username and password are used in a subsequent Binding Request and Binding Response, for the purposes of authentication and message integrity. 
Binding requests are used to determine the bindings allocated by NATs. The client sends a Binding Request to the server, over UDP. The server examines the source IP address and port of the request, and copies them into a response that is sent back to the client. There are some parameters in the request that allow the client to ask that the response be sent elsewhere, or that the server send the response from a different address and port. There are attributes for providing message integrity and authentication.

The trick is using STUN to discover the presence of NAT, and to learn and use the bindings they allocate.

The STUN client is typically embedded in an application which needs to obtain a public IP address and port that can be used to receive data. For example, it might need to obtain an IP address and port to receive Real Time Transport Protocol (RTP) [12] traffic. When the application starts, the STUN client within the application sends a STUN Shared Secret Request to its server, obtains a username and password, and then sends it a Binding Request. STUN servers can be discovered through DNS SRV records [3], and it is generally assumed that the client is configured with the domain to use to find the STUN server. Generally, this will be the domain of the provider of the service the application is using (such a provider is incented to deploy STUN servers in order to allow its customers to use its application through $\mathrm{NAT}$ ). Of course, a client can determine the address or domain name of a STUN server through other means. A STUN server can even be embedded within an end system.

The STUN Binding Request is used to discover the presence of a NAT, and to discover the public IP address and port mappings generated by the NAT. Binding Requests are sent to the STUN server using UDP. When a Binding Request arrives at the STUN server, it may have passed through one or more NATs between the STUN client and the STUN server. As a result, the source address of the request received by the server will be the mapped address created by the NAT closest to the server. The STUN server copies that source IP address and port into a STUN Binding Response, and sends it back to the source IP address and port of the STUN request. For all of the NAT types above, this response will arrive at the STUN client.

When the STUN client receives the STUN Binding Response, it compares the IP address and port in the packet with the local IP address and port it bound to when the request was sent. If these do not match, the STUN client is behind one or more NATs. In the case of a fullcone NAT, the IP address and port in the body of the STUN response are public, and can be used by any host on the public Internet to send packets to the application that sent the STUN request. An application need only listen on the IP address and port from which 
the STUN request was sent. Any packets sent by a host on the public Internet to the public address and port learned by STUN will be received by the application.

Of course, the host may not be behind a full-cone NAT. Indeed, it doesn't yet know what type of NAT it is behind. To determine that, the client uses additional STUN Binding Requests. The exact procedure is flexible, but would generally work as follows. The client would send a second STUN Binding Request, this time to a different IP address, but from the same source IP address and port. If the IP address and port in the response are different from those in the first response, the client knows it is behind a symmetric NAT. To determine if it's behind a full-cone NAT, the client can send a STUN Binding Request with flags that tell the STUN server to send a response from a different IP address and port than the request was received on. In other words, if the client sent a Binding Request to IP address/port $A / B$ using a source IP address/port of $X / Y$, the STUN server would send the Binding Response to X/Y using source IP address/port C/D. If the client receives this response, it knows it is behind a full cone NAT.

STUN also allows the client to ask the server to send the Binding Response from the same IP address the request was received on, but with a different port. This can be used to detect whether the client is behind a port restricted cone NAT or just a restricted cone NAT.

It should be noted that the configuration in Figure 1 is not the only permissible configuration. The STUN server can be located anywhere, including within another client. The only requirement is that the STUN server is reachable by the client, and if the client is trying to obtain a publicly routable address, that the server reside on the public Internet.

\section{Message Overview}

STUN messages are TLV (type-length-value) encoded using big endian (network ordered) binary. All STUN messages start with a STUN header, followed by a STUN payload. The payload is a series of STUN attributes, the set of which depends on the message type. The STUN header contains a STUN message type, transaction ID, and length. The message type can be Binding Request, Binding Response, Binding Error Response, Shared Secret Request, Shared Secret Response, or Shared Secret Error Response. The transaction ID is used to correlate requests and responses. The length indicates the total length of the STUN payload, not including the header. This allows STUN to run over TCP. Shared Secret Requests are always sent over TCP (indeed, using TLS over TCP). 
Several STUN attributes are defined. The first is a MAPPED-ADDRESS attribute, which is an IP address and port. It is always placed in the Binding Response, and it indicates the source IP address and port the server saw in the Binding Request. There is also a RESPONSEADDRESS attribute, which contains an IP address and port. The RESPONSE-ADDRESS attribute can be present in the Binding Request, and indicates where the Binding Response is to be sent. It's optional, and when not present, the Binding Response is sent to the source IP address and port of the Binding Request.

The third attribute is the CHANGE-REQUEST attribute, and it contains two flags to control the IP address and port used to send the response. These flags are called "change IP" and "change port" flags. The CHANGE-REQUEST attribute is allowed only in the Binding Request. The "change IP" and "change port" flags are useful for determining whether the client is behind a restricted cone NAT or restricted port cone NAT. They instruct the server to send the Binding Responses from a different source IP address and port. The CHANGE-REQUEST attribute is optional in the Binding Request.

The fourth attribute is the CHANGED-ADDRESS attribute. It is present in Binding Responses. It informs the client of the source IP address and port that would be used if the client requested the "change IP" and "change port" behavior.

The fifth attribute is the SOURCE-ADDRESS attribute. It is only present in Binding Responses. It indicates the source IP address and port where the response was sent from. It is useful for detecting twice NAT configurations.

The sixth attribute is the USERNAME attribute. It is present in a Shared Secret Response, which provides the client with a temporary username and password (encoded in the PASSWORD attribute). The USERNAME is also present in Binding Requests, serving as an index to the shared secret used for the integrity protection of the Binding Request. The seventh attribute, PASSWORD, is only found in Shared Secret Response messages. The eight attribute is the MESSAGEINTEGRITY attribute, which contains a message integrity check over the Binding Request or Binding Response.

The ninth attribute is the ERROR-CODE attribute. This is present in the Binding Error Response and Shared Secret Error Response. It indicates the error that has occurred. The tenth attribute is the UNKNOWN-ATTRIBUTES attribute, which is present in either the Binding Error Response or Shared Secret Error Response. It indicates the mandatory attributes from the request which were unknown. The eleventh attribute is the REFLECTED-FROM attribute, which is present in Binding Responses. It indicates the IP address and port of the 
sender of a Binding Request, used for traceability purposes to prevent certain denial-of-service attacks.

8. Server Behavior

The server behavior depends on whether the request is a Binding Request or a Shared Secret Request.

\subsection{Binding Requests}

A STUN server MUST be prepared to receive Binding Requests on four address/port combinations - (A1, P1), (A2, P1), (A1, P2), and (A2, P2). (A1, P1) represent the primary address and port, and these are the ones obtained through the client discovery procedures below. Typically, P1 will be port 3478, the default STUN port. A2 and P2 are arbitrary. A2 and P2 are advertised by the server through the CHANGED-ADDRESS attribute, as described below.

It is RECOMMENDED that the server check the Binding Request for a MESSAGE-INTEGRITY attribute. If not present, and the server requires integrity checks on the request, it generates a Binding Error Response with an ERROR-CODE attribute with response code 401 . If the MESSAGE-INTEGRITY attribute was present, the server computes the HMAC over the request as described in section 11.2.8. The key to use depends on the shared secret mechanism. If the STUN Shared Secret Request was used, the key MUST be the one associated with the USERNAME attribute present in the request. If the USERNAME attribute was not present, the server MUST generate a Binding Error Response. The Binding Error Response MUST include an ERROR-CODE attribute with response code 432. If the USERNAME is present, but the server doesn't remember the shared secret for that USERNAME (because it timed out, for example), the server MUST generate a Binding Error Response. The Binding Error Response MUST include an ERROR-CODE attribute with response code 430. If the server does know the shared secret, but the computed HMAC differs from the one in the request, the server MUST generate a Binding Error Response with an ERROR-CODE attribute with response code 431. The Binding Error Response is sent to the IP address and port the Binding Request came from, and sent from the IP address and port the Binding Request was sent to.

Assuming the message integrity check passed, processing continues. The server MUST check for any attributes in the request with values less than or equal to 0x7fff which it does not understand. If it encounters any, the server MUST generate a Binding Error Response, and it MUST include an ERROR-CODE attribute with a 420 response code. 
That response MUST contain an UNKNOWN-ATTRIBUTES attribute listing the attributes with values less than or equal to 0x7fff which were not understood. The Binding Error Response is sent to the IP address and port the Binding Request came from, and sent from the IP address and port the Binding Request was sent to.

Assuming the request was correctly formed, the server MUST generate a single Binding Response. The Binding Response MUST contain the same transaction ID contained in the Binding Request. The length in the message header MUST contain the total length of the message in bytes, excluding the header. The Binding Response MUST have a message type of "Binding Response".

The server MUST add a MAPPED-ADDRESS attribute to the Binding Response. The IP address component of this attribute MUST be set to the source IP address observed in the Binding Request. The port component of this attribute MUST be set to the source port observed in the Binding Request.

If the RESPONSE-ADDRESS attribute was absent from the Binding Request, the destination address and port of the Binding Response MUST be the same as the source address and port of the Binding Request. Otherwise, the destination address and port of the Binding Response MUST be the value of the IP address and port in the RESPONSE-ADDRESS attribute.

The source address and port of the Binding Response depend on the value of the CHANGE-REQUEST attribute and on the address and port the Binding Request was received on, and are summarized in Table 1.

Let Da represent the destination IP address of the Binding Request (which will be either A1 or A2), and Dp represent the destination port of the Binding Request (which will be either P1 or P2). Let Ca represent the other address, so that if Da is A1, Ca is A2. If Da is $A 2, C a$ is $A 1$. Similarly, let $C p$ represent the other port, so that if $\mathrm{Dp}$ is $\mathrm{P} 1, \mathrm{Cp}$ is $\mathrm{P} 2$. If $\mathrm{Dp}$ is $\mathrm{P} 2, \mathrm{Cp}$ is $\mathrm{P} 1$. If the "change port" flag was set in CHANGE-REQUEST attribute of the Binding Request, and the "change IP" flag was not set, the source IP address of the Binding Response MUST be Da and the source port of the Binding Response MUST be Cp. If the "change IP" flag was set in the Binding Request, and the "change port" flag was not set, the source IP address of the Binding Response MUST be Ca and the source port of the Binding Response MUST be Dp. When both flags are set, the source IP address of the Binding Response MUST be $\mathrm{Ca}$ and the source port of the Binding Response MUST be Cp. If neither flag is set, or if the CHANGE-REQUEST attribute is absent entirely, the source IP address of the Binding Response MUST be Da and the source port of the Binding Response MUST be Dp. 


$\begin{array}{llll}\text { Flags } & \text { Source Address } & \text { Source Port } & \text { CHANGED-ADDRESS } \\ \text { none } & \mathrm{Da} & \mathrm{Dp} & \mathrm{Ca}: \mathrm{Cp} \\ \text { Change IP } & \mathrm{Ca} & \mathrm{Dp} & \mathrm{Ca}: \mathrm{Cp} \\ \text { Change port } & \mathrm{Da} & \mathrm{Cp} & \mathrm{Ca}: \mathrm{Cp} \\ \begin{array}{l}\text { Change IP and } \\ \quad\end{array} & & \mathrm{Ca}: \mathrm{Cp}\end{array}$

Table 1: Impact of Flags on Packet Source and CHANGED-ADDRESS

The server MUST add a SOURCE-ADDRESS attribute to the Binding Response, containing the source address and port used to send the Binding Response.

The server MUST add a CHANGED-ADDRESS attribute to the Binding Response. This contains the source IP address and port that would be used if the client had set the "change IP" and "change port" flags in the Binding Request. As summarized in Table 1, these are Ca and Cp, respectively, regardless of the value of the CHANGE-REQUEST flags.

If the Binding Request contained both the USERNAME and MESSAGEINTEGRITY attributes, the server MUST add a MESSAGE-INTEGRITY attribute to the Binding Response. The attribute contains an HMAC [13] over the response, as described in section 11.2.8. The key to use depends on the shared secret mechanism. If the STUN Shared Secret Request was used, the key MUST be the one associated with the USERNAME attribute present in the Binding Request.

If the Binding Request contained a RESPONSE-ADDRESS attribute, the server MUST add a REFLECTED-FROM attribute to the response. If the Binding Request was authenticated using a username obtained from a Shared Secret Request, the REFLECTED-FROM attribute MUST contain the source IP address and port where that Shared Secret Request came from. If the username present in the request was not allocated using a Shared Secret Request, the REFLECTED-FROM attribute MUST contain the source address and port of the entity which obtained the username, as best can be verified with the mechanism used to allocate the username. If the username was not present in the request, and the server was willing to process the request, the REFLECTED-FROM attribute SHOULD contain the source IP address and port where the request came from.

The server SHOULD NOT retransmit the response. Reliability is achieved by having the client periodically resend the request, each of which triggers a response from the server. 


\subsection{Shared Secret Requests}

Shared Secret Requests are always received on TLS connections. When the server receives a request from the client to establish a TLS connection, it MUST proceed with TLS, and SHOULD present a site certificate. The TLS ciphersuite TLS_RSA_WITH_AES_128_CBC_SHA [4] SHOULD be used. Client TLS authentication MUST NOT be done, since the server is not allocating any resources to clients, and the computational burden can be a source of attacks.

If the server receives a Shared Secret Request, it MUST verify that the request arrived on a TLS connection. If it did not receive the request over TLS, it MUST generate a Shared Secret Error Response, and it MUST include an ERROR-CODE attribute with a 433 response code. The destination for the error response depends on the transport on which the request was received. If the Shared secret Request was received over TCP, the Shared Secret Error Response is sent over the same connection the request was received on. If the shared secret Request was receive over UDP, the Shared Secret Error Response is sent to the source IP address and port that the request came from.

The server MUST check for any attributes in the request with values less than or equal to 0x7fff which it does not understand. If it encounters any, the server MUST generate a shared Secret Error Response, and it MUST include an ERROR-CODE attribute with a 420 response code. That response MUST contain an UNKNOWN-ATTRIBUTES attribute listing the attributes with values less than or equal to 0x7fff which were not understood. The Shared Secret Error Response is sent over the TLS connection.

All Shared Secret Error Responses MUST contain the same transaction ID contained in the Shared Secret Request. The length in the message header MUST contain the total length of the message in bytes, excluding the header. The Shared Secret Error Response MUST have a message type of "Shared Secret Error Response" (0x0112).

Assuming the request was properly constructed, the server creates a Shared Secret Response. The Shared Secret Response MUST contain the same transaction ID contained in the shared Secret Request. The length in the message header MUST contain the total length of the message in bytes, excluding the header. The Shared Secret Response MUST have a message type of "Shared Secret Response". The Shared Secret Response MUST contain a USERNAME attribute and a PASSWORD attribute. The USERNAME attribute serves as an index to the password, which is contained in the PASSWORD attribute. The server can use any mechanism it chooses to generate the username. However, the username MUST be valid for a period of at least 10 minutes. Validity means that the server can compute the password for that 
username. There MUST be a single password for each username. In other words, the server cannot, 10 minutes later, assign a different password to the same username. The server MUST hand out a different username for each distinct shared secret Request. Distinct, in this case, implies a different transaction ID. It is RECOMMENDED that the server explicitly invalidate the username after ten minutes. It MUST invalidate the username after 30 minutes. The PASSWORD contains the password bound to that username. The password MUST have at least 128 bits. The likelihood that the server assigns the same password for two different usernames MUST be vanishingly small, and the passwords MUST be unguessable. In other words, they MUST be a cryptographically random function of the username.

These requirements can still be met using a stateless server, by intelligently computing the USERNAME and PASSWORD. One approach is to construct the USERNAME as:

USERNAME = <prefix, rounded-time, clientIP, hmac>

Where prefix is some random text string (different for each shared secret request), rounded-time is the current time modulo 20 minutes, clientIP is the source IP address where the shared secret Request came from, and hmac is an HMAC [13] over the prefix, rounded-time, and client IP, using a server private key.

The password is then computed as:

password = <hmac (USERNAME, anotherprivatekey) $>$

With this structure, the username itself, which will be present in the Binding Request, contains the source IP address where the Shared Secret Request came from. That allows the server to meet the requirements specified in section 8.1 for constructing the REFLECTED-FROM attribute. The server can verify that the username was not tampered with, using the hmac present in the username.

The Shared Secret Response is sent over the same TLS connection the request was received on. The server SHOULD keep the connection open, and let the client close it.

9. Client Behavior

The behavior of the client is very straightforward. Its task is to discover the STUN server, obtain a shared secret, formulate the Binding Request, handle request reliability, and process the Binding Responses. 


\subsection{Discovery}

Generally, the client will be configured with a domain name of the provider of the STUN servers. This domain name is resolved to an IP address and port using the SRV procedures specified in RFC 2782 [3].

Specifically, the service name is "stun". The protocol is "udp" for sending Binding Requests, or "tcp" for sending Shared Secret Requests. The procedures of RFC 2782 are followed to determine the server to contact. RFC 2782 spells out the details of how a set of SRV records are sorted and then tried. However, it only states that the client should "try to connect to the (protocol, address, service)" without giving any details on what happens in the event of failure. Those details are described here for STUN.

For STUN requests, failure occurs if there is a transport failure of some sort (generally, due to fatal ICMP errors in UDP or connection failures in TCP). Failure also occurs if the transaction fails due to timeout. This occurs 9.5 seconds after the first request is sent, for both Shared Secret Requests and Binding Requests. See Section 9.3 for details on transaction timeouts for Binding Requests. If a failure occurs, the client SHOULD create a new request, which is identical to the previous, but has a different transaction ID and MESSAGE INTEGRITY attribute (the HMAC will change because the transaction ID has changed). That request is sent to the next element in the list as specified by RFC 2782 .

The default port for STUN requests is 3478, for both TCP and UDP. Administrators SHOULD use this port in their SRV records, but MAY use others.

If no SRV records were found, the client performs an A record lookup of the domain name. The result will be a list of IP addresses, each of which can be contacted at the default port.

This would allow a firewall admin to open the STUN port, so hosts within the enterprise could access new applications. Whether they will or won't do this is a good question.

\subsection{Obtaining a shared Secret}

As discussed in Section 12, there are several attacks possible on STUN systems. Many of these are prevented through integrity of requests and responses. To provide that integrity, sTUN makes use of a shared secret between client and server, used as the keying material for an HMAC used in both the Binding Request and Binding Response. STUN allows for the shared secret to be obtained in any way (for example, Kerberos [14]). However, it MUST have at least 128 
bits of randomness. In order to ensure interoperability, this specification describes a TLS-based mechanism. This mechanism, described in this section, MUST be implemented by clients and servers.

First, the client determines the IP address and port that it will open a TCP connection to. This is done using the discovery procedures in section 9.1. The client opens up the connection to that address and port, and immediately begins TLS negotiation [2]. The client MUST verify the identity of the server. To do that, it follows the identification procedures defined in section 3.1 of RFC 2818 [5]. Those procedures assume the client is dereferencing a URI. For purposes of usage with this specification, the client treats the domain name or IP address used in Section 9.1 as the host portion of the URI that has been dereferenced.

Once the connection is opened, the client sends a shared Secret request. This request has no attributes, just the header. The transaction ID in the header MUST meet the requirements outlined for the transaction ID in a binding request, described in section 9.3 below. The server generates a response, which can either be a shared Secret Response or a Shared Secret Error Response.

If the response was a Shared Secret Error Response, the client checks the response code in the ERROR-CODE attribute. Interpretation of those response codes is identical to the processing of section 9.4 for the Binding Error Response.

If a client receives a Shared Secret Response with an attribute whose type is greater than 0x7fff, the attribute MUST be ignored. If the client receives a shared Secret Response with an attribute whose type is less than or equal to 0x7fff, the response is ignored.

If the response was a Shared Secret Response, it will contain a short lived username and password, encoded in the USERNAME and PASSWORD attributes, respectively.

The client MAY generate multiple shared Secret Requests on the connection, and it MAY do so before receiving shared secret Responses to previous Shared Secret Requests. The client SHOULD close the connection as soon as it has finished obtaining usernames and passwords.

Section 9.3 describes how these passwords are used to provide integrity protection over Binding Requests, and Section 8.1 describes how it is used in Binding Responses. 


\subsection{Formulating the Binding Request}

A Binding Request formulated by the client follows the syntax rules defined in section 11. Any two requests that are not bit-wise identical, and not sent to the same server from the same IP address and port, MUST carry different transaction IDs. The transaction ID MUST be uniformly and randomly distributed between 0 and $2 * \star 128-1$. The large range is needed because the transaction ID serves as a form of randomization, helping to prevent replays of previously signed responses from the server. The message type of the request MUST be "Binding Request".

The RESPONSE-ADDRESS attribute is optional in the Binding Request. It is used if the client wishes the response to be sent to a different IP address and port than the one the request was sent from. This is useful for determining whether the client is behind a firewall, and for applications that have separated control and data components. See Section 10.3 for more details. The CHANGE-REQUEST attribute is also optional. Whether it is present depends on what the application is trying to accomplish. See Section 10 for some example uses.

The client SHOULD add a MESSAGE-INTEGRITY and USERNAME attribute to the Binding Request. This MESSAGE-INTEGRITY attribute contains an HMAC [13]. The value of the username, and the key to use in the MESSAGE-INTEGRITY attribute depend on the shared secret mechanism. If the STUN Shared Secret Request was used, the USERNAME must be a valid username obtained from a shared Secret Response within the last nine minutes. The shared secret for the HMAC is the value of the PASSWORD attribute obtained from the same shared Secret Response.

Once formulated, the client sends the Binding Request. Reliability is accomplished through client retransmissions. Clients SHOULD retransmit the request starting with an interval of $100 \mathrm{~ms}$, doubling every retransmit until the interval reaches 1.6s. Retransmissions continue with intervals of $1.6 \mathrm{~s}$ until a response is received, or a total of 9 requests have been sent. If no response is received by 1.6 seconds after the last request has been sent, the client SHOULD consider the transaction to have failed. In other words, requests would be sent at times 0ms, 100ms, 300ms, $700 \mathrm{~ms}, 1500 \mathrm{~ms}, 3100 \mathrm{~ms}$, $4700 \mathrm{~ms}, 6300 \mathrm{~ms}$, and $7900 \mathrm{~ms}$. At $9500 \mathrm{~ms}$, the client considers the transaction to have failed if no response has been received.

\subsection{Processing Binding Responses}

The response can either be a Binding Response or Binding Error Response. Binding Error Responses are always received on the source address and port the request was sent from. A Binding Response will 
be received on the address and port placed in the RESPONSE-ADDRESS attribute of the request. If none was present, the Binding Responses will be received on the source address and port the request was sent from.

If the response is a Binding Error Response, the client checks the response code from the ERROR-CODE attribute of the response. For a 400 response code, the client SHOULD display the reason phrase to the user. For a 420 response code, the client sHOULD retry the request, this time omitting any attributes listed in the UNKNOWN-ATTRIBUTES attribute of the response. For a 430 response code, the client SHOULD obtain a new shared secret, and retry the Binding Request with a new transaction. For 401 and 432 response codes, if the client had omitted the USERNAME or MESSAGE-INTEGRITY attribute as indicated by the error, it SHOULD try again with those attributes. For a 431 response code, the client SHOULD alert the user, and MAY try the request again after obtaining a new username and password. For a 500 response code, the client MAY wait several seconds and then retry the request. For a 600 response code, the client MUST NOT retry the request, and SHOULD display the reason phrase to the user. Unknown attributes between 400 and 499 are treated like a 400, unknown attributes between 500 and 599 are treated like a 500, and unknown attributes between 600 and 699 are treated like a 600. Any response between 100 and 399 MUST result in the cessation of request retransmissions, but otherwise is discarded.

If a client receives a response with an attribute whose type is greater than 0x7fff, the attribute MUST be ignored. If the client receives a response with an attribute whose type is less than or equal to 0x7fff, request retransmissions MUST cease, but the entire response is otherwise ignored.

If the response is a Binding Response, the client SHOULD check the response for a MESSAGE-INTEGRITY attribute. If not present, and the client placed a MESSAGE-INTEGRITY attribute into the request, it MUST discard the response. If present, the client computes the HMAC over the response as described in section 11.2.8. The key to use depends on the shared secret mechanism. If the STUN Shared Secret Request was used, the key MUST be same as used to compute the MESSAGEINTEGRITY attribute in the request. If the computed HMAC differs from the one in the response, the client MUST discard the response, and SHOULD alert the user about a possible attack. If the computed HMAC matches the one from the response, processing continues.

Reception of a response (either Binding Error Response or Binding Response) to a Binding Request will terminate retransmissions of that request. However, clients MUST continue to listen for responses to a Binding Request for 10 seconds after the first response. If it 
receives any responses in this interval with different message types (Binding Responses and Binding Error Responses, for example) or different MAPPED-ADDRESSes, it is an indication of a possible attack. The client MUST NOT use the MAPPED-ADDRESS from any of the responses it received (either the first or the additional ones), and SHOULD alert the user.

Furthermore, if a client receives more than twice as many Binding Responses as the number of Binding Requests it sent, it MUST NOT use the MAPPED-ADDRESS from any of those responses, and SHOULD alert the user about a potential attack.

If the Binding Response is authenticated, and the MAPPED-ADDRESS was not discarded because of a potential attack, the CLIENT MAY use the MAPPED-ADDRESS and SOURCE-ADDRESS attributes.

10. Use Cases

The rules of Sections 8 and 9 describe exactly how a client and server interact to send requests and get responses. However, they do not dictate how the STUN protocol is used to accomplish useful tasks. That is at the discretion of the client. Here, we provide some useful scenarios for applying STUN.

\subsection{Discovery Process}

In this scenario, a user is running a multimedia application which needs to determine which of the following scenarios applies to it:

o On the open Internet

- Firewall that blocks UDP

- Firewall that allows UDP out, and responses have to come back to the source of the request (like a symmetric NAT, but no translation. We call this a symmetric UDP Firewall)

- Full-cone NAT

- Symmetric NAT

- Restricted cone or restricted port cone NAT

Which of the six scenarios applies can be determined through the flow chart described in Figure 2. The chart refers only to the sequence of Binding Requests; Shared Secret Requests will, of course, be needed to authenticate each Binding Request used in the sequence. 
The flow makes use of three tests. In test I, the client sends a STUN Binding Request to a server, without any flags set in the CHANGE-REQUEST attribute, and without the RESPONSE-ADDRESS attribute. This causes the server to send the response back to the address and port that the request came from. In test II, the client sends a Binding Request with both the "change IP" and "change port" flags from the CHANGE-REQUEST attribute set. In test III, the client sends a Binding Request with only the "change port" flag set.

The client begins by initiating test I. If this test yields no response, the client knows right away that it is not capable of UDP connectivity. If the test produces a response, the client examines the MAPPED-ADDRESS attribute. If this address and port are the same as the local IP address and port of the socket used to send the request, the client knows that it is not natted. It executes test II.

If a response is received, the client knows that it has open access to the Internet (or, at least, its behind a firewall that behaves like a full-cone NAT, but without the translation). If no response is received, the client knows its behind a symmetric UDP firewall.

In the event that the IP address and port of the socket did not match the MAPPED-ADDRESS attribute in the response to test $I$, the client knows that it is behind a NAT. It performs test II. If a response is received, the client knows that it is behind a full-cone NAT. If no response is received, it performs test I again, but this time, does so to the address and port from the CHANGED-ADDRESS attribute from the response to test I. If the IP address and port returned in the MAPPED-ADDRESS attribute are not the same as the ones from the first test $I$, the client knows its behind a symmetric NAT. If the address and port are the same, the client is either behind a restricted or port restricted NAT. To make a determination about which one it is behind, the client initiates test III. If a response is received, its behind a restricted NAT, and if no response is received, its behind a port restricted NAT.

This procedure yields substantial information about the operating condition of the client application. In the event of multiple NATs between the client and the Internet, the type that is discovered will be the type of the most restrictive NAT between the client and the Internet. The types of NAT, in order of restrictiveness, from most to least, are symmetric, port restricted cone, restricted cone, and full cone.

Typically, a client will re-do this discovery process periodically to detect changes, or look for inconsistent results. It is important to note that when the discovery process is redone, it should not 
generally be done from the same local address and port used in the previous discovery process. If the same local address and port are reused, bindings from the previous test may still be in existence, and these will invalidate the results of the test. Using a different local address and port for subsequent tests resolves this problem. An alternative is to wait sufficiently long to be confident that the old bindings have expired (half an hour should more than suffice).

10.2 Binding Lifetime Discovery

STUN can also be used to discover the lifetimes of the bindings created by the NAT. In many cases, the client will need to refresh the binding, either through a new STUN request, or an application packet, in order for the application to continue to use the binding. By discovering the binding lifetime, the client can determine how frequently it needs to refresh. 


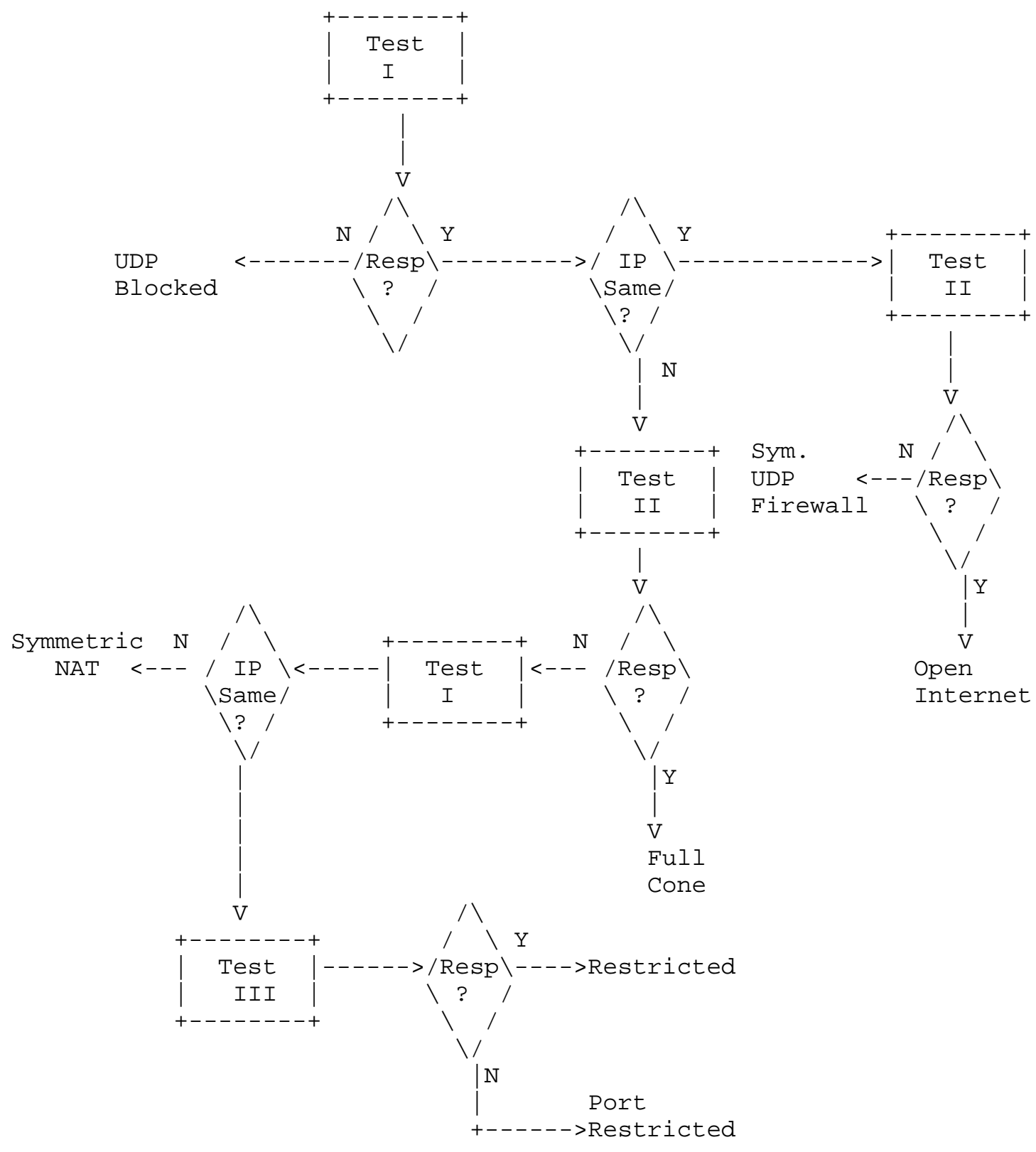

Figure 2: Flow for type discovery process 
To determine the binding lifetime, the client first sends a Binding Request to the server from a particular socket, $X$. This creates a binding in the NAT. The response from the server contains a MAPPEDADDRESS attribute, providing the public address and port on the NAT. Call this $\mathrm{Pa}$ and $\mathrm{Pp}$, respectively. The client then starts a timer with a value of $\mathrm{T}$ seconds. When this timer fires, the client sends another Binding Request to the server, using the same destination address and port, but from a different socket, $Y$. This request contains a RESPONSE-ADDRESS address attribute, set to (Pa,Pp). This will create a new binding on the NAT, and cause the STUN server to send a Binding Response that would match the old binding, if it still exists. If the client receives the Binding Response on socket $x$, it knows that the binding has not expired. If the client receives the Binding Response on socket $Y$ (which is possible if the old binding expired, and the NAT allocated the same public address and port to the new binding), or receives no response at all, it knows that the binding has expired.

The client can find the value of the binding lifetime by doing a binary search through $\mathrm{T}$, arriving eventually at the value where the response is not received for any timer greater than $\mathrm{T}$, but is received for any timer less than $\mathrm{T}$.

This discovery process takes quite a bit of time, and is something that will typically be run in the background on a device once it boots.

It is possible that the client can get inconsistent results each time this process is run. For example, if the NAT should reboot, or be reset for some reason, the process may discover a lifetime than is shorter than the actual one. For this reason, implementations are encouraged to run the test numerous times, and be prepared to get inconsistent results.

\subsection{Binding Acquisition}

Consider once more the case of a VoIP phone. It used the discovery process above when it started up, to discover its environment. Now, it wants to make a call. As part of the discovery process, it determined that it was behind a full-cone NAT.

Consider further that this phone consists of two logically separated components - a control component that handles signaling, and a media component that handles the audio, video, and RTP [12]. Both are behind the same NAT. Because of this separation of control and media, we wish to minimize the communication required between them. In fact, they may not even run on the same host. 
In order to make a voice call, the phone needs to obtain an IP address and port that it can place in the call setup message as the destination for receiving audio.

To obtain an address, the control component sends a Shared Secret Request to the server, obtains a shared secret, and then sends a Binding Request to the server. No CHANGE-REQUEST attribute is present in the Binding Request, and neither is the RESPONSE-ADDRESS attribute. The Binding Response contains a mapped address. The control component then formulates a second Binding Request. This request contains a RESPONSE-ADDRESS, which is set to the mapped address learned from the previous Binding Response. This Binding Request is passed to the media component, along with the IP address and port of the STUN server. The media component sends the Binding Request. The request goes to the STUN server, which sends the Binding Response back to the control component. The control component receives this, and now has learned an IP address and port that will be routed back to the media component that sent the request.

The client will be able to receive media from anywhere on this mapped address.

In the case of silence suppression, there may be periods where the client receives no media. In this case, the UDP bindings could timeout (UDP bindings in NATs are typically short; 30 seconds is common). To deal with this, the application can periodically retransmit the query in order to keep the binding fresh.

It is possible that both participants in the multimedia session are behind the same NAT. In that case, both will repeat this procedure above, and both will obtain public address bindings. When one sends media to the other, the media is routed to the NAT, and then turns right back around to come back into the enterprise, where it is translated to the private address of the recipient. This is not particularly efficient, and unfortunately, does not work in many commercial NATs. In such cases, the clients may need to retry using private addresses.

11. Protocol Details

This section presents the detailed encoding of a STUN message.

STUN is a request-response protocol. Clients send a request, and the server sends a response. There are two requests, Binding Request, and Shared Secret Request. The response to a Binding Request can 
either be the Binding Response or Binding Error Response. The response to a Shared Secret Request can either be a Shared Secret Response or a Shared Secret Error Response.

STUN messages are encoded using binary fields. All integer fields are carried in network byte order, that is, most significant byte (octet) first. This byte order is commonly known as big-endian. The transmission order is described in detail in Appendix B of RFC 791

[6]. Unless otherwise noted, numeric constants are in decimal (base 10).

\subsection{Message Header}

All STUN messages consist of a 20 byte header:

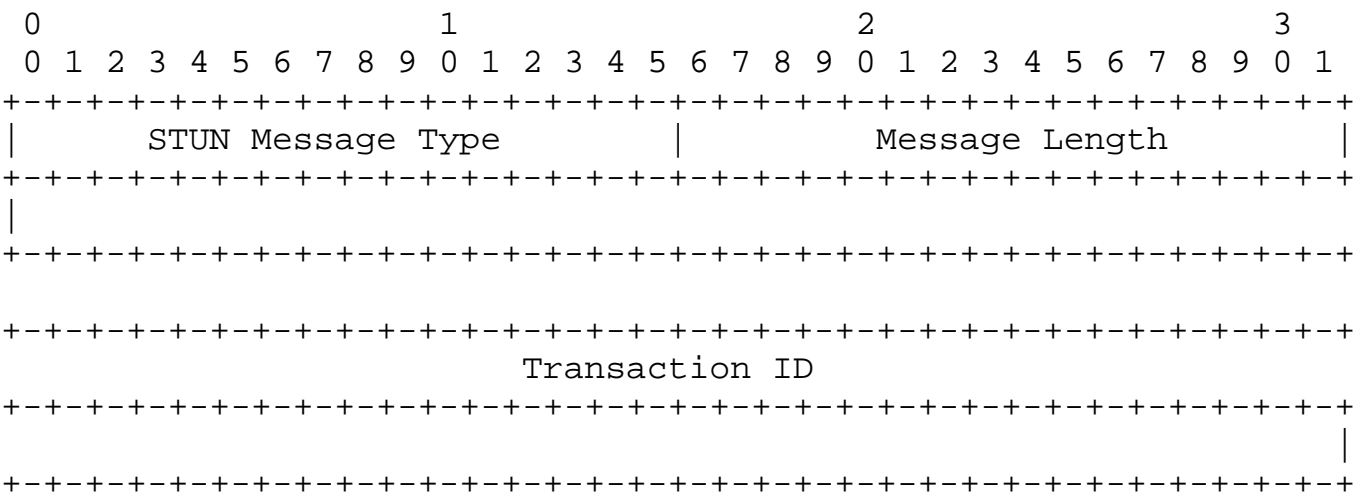

The Message Types can take on the following values:

0x0001 $:$ Binding Request
0x0101 $:$ Binding Response
0x0111 : Binding Error Response
0x0002 $:$ Shared Secret Request
0x0102 : Shared Secret Response
0x0112 : Shared Secret Error Response

The message length is the count, in bytes, of the size of the message, not including the 20 byte header.

The transaction ID is a 128 bit identifier. It also serves as salt to randomize the request and the response. All responses carry the same identifier as the request they correspond to. 


\subsection{Message Attributes}

After the header are 0 or more attributes. Each attribute is TLV encoded, with a 16 bit type, 16 bit length, and variable value:

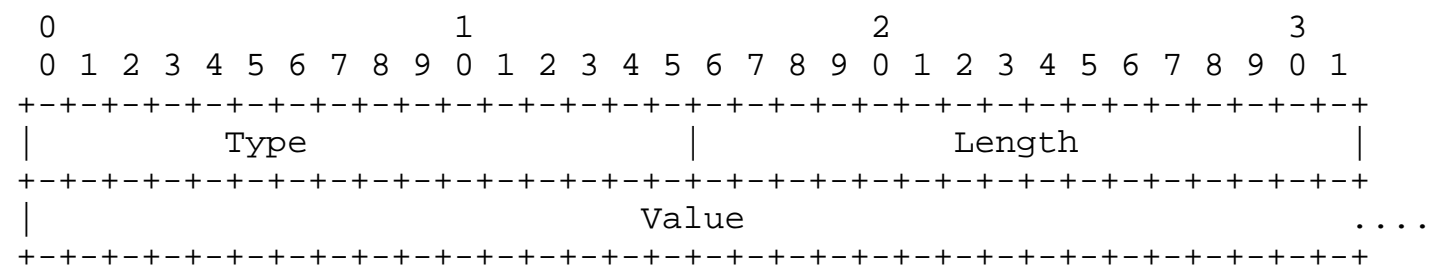

The following types are defined:

0X0001: MAPPED-ADDRESS

0x0002: RESPONSE-ADDRESS

0x0003: CHANGE-REQUEST

0x0004: SOURCE-ADDRESS

0x0005: CHANGED-ADDRESS

0X0006: USERNAME

0x0007: PASSWORD

0x0008: MESSAGE-INTEGRITY

0X0009: ERROR-CODE

0x000a: UNKNOWN-ATTRIBUTES

0x000b: REFLECTED-FROM

To allow future revisions of this specification to add new attributes if needed, the attribute space is divided into optional and mandatory ones. Attributes with values greater than 0x7fff are optional, which means that the message can be processed by the client or server even though the attribute is not understood. Attributes with values less than or equal to $0 x 7 f f f$ are mandatory to understand, which means that the client or server cannot process the message unless it understands the attribute.

The MESSAGE-INTEGRITY attribute MUST be the last attribute within a message. Any attributes that are known, but are not supposed to be present in a message (MAPPED-ADDRESS in a request, for example) MUST be ignored.

Table 2 indicates which attributes are present in which messages. An $M$ indicates that inclusion of the attribute in the message is mandatory, O means its optional, C means it's conditional based on some other aspect of the message, and N/A means that the attribute is not applicable to that message type. 


\begin{tabular}{|c|c|c|c|c|c|c|}
\hline Att. & $\begin{array}{l}\text { Binding } \\
\text { Req. }\end{array}$ & $\begin{array}{l}\text { Binding } \\
\text { Resp. }\end{array}$ & $\begin{array}{l}\text { Binding } \\
\text { Error } \\
\text { Resp. }\end{array}$ & $\begin{array}{l}\text { Shared } \\
\text { Secret } \\
\text { Req. }\end{array}$ & $\begin{array}{l}\text { Shared } \\
\text { Secret } \\
\text { Resp. }\end{array}$ & $\begin{array}{l}\text { Shared } \\
\text { Secret } \\
\text { Error } \\
\text { Resp. }\end{array}$ \\
\hline MAPPED-ADDRESS & $\mathrm{N} / \mathrm{A}$ & $\mathrm{M}$ & $\mathrm{N} / \mathrm{A}$ & $\mathrm{N} / \mathrm{A}$ & $\mathrm{N} / \mathrm{A}$ & $\mathrm{N} / \mathrm{A}$ \\
\hline RESPONSE-ADDRESS & 0 & $\mathrm{~N} / \mathrm{A}$ & $\mathrm{N} / \mathrm{A}$ & $\mathrm{N} / \mathrm{A}$ & $\mathrm{N} / \mathrm{A}$ & $\mathrm{N} / \mathrm{A}$ \\
\hline CHANGE-REQUEST & 0 & $\mathrm{~N} / \mathrm{A}$ & $\mathrm{N} / \mathrm{A}$ & $\mathrm{N} / \mathrm{A}$ & $\mathrm{N} / \mathrm{A}$ & $\mathrm{N} / \mathrm{A}$ \\
\hline SOURCE-ADDRESS & $\mathrm{N} / \mathrm{A}$ & M & $\mathrm{N} / \mathrm{A}$ & $\mathrm{N} / \mathrm{A}$ & $\mathrm{N} / \mathrm{A}$ & $\mathrm{N} / \mathrm{A}$ \\
\hline CHANGED-ADDRESS & $\mathrm{N} / \mathrm{A}$ & $\mathrm{M}$ & $\mathrm{N} / \mathrm{A}$ & $\mathrm{N} / \mathrm{A}$ & $\mathrm{N} / \mathrm{A}$ & $\mathrm{N} / \mathrm{A}$ \\
\hline USERNAME & $\mathrm{O}$ & $\mathrm{N} / \mathrm{A}$ & $\mathrm{N} / \mathrm{A}$ & $\mathrm{N} / \mathrm{A}$ & M & $\mathrm{N} / \mathrm{A}$ \\
\hline PASSWORD & $\mathrm{N} / \mathrm{A}$ & $\mathrm{N} / \mathrm{A}$ & $\mathrm{N} / \mathrm{A}$ & $\mathrm{N} / \mathrm{A}$ & M & $\mathrm{N} / \mathrm{A}$ \\
\hline MESSAGE-INTEGRITY & 0 & 0 & $\mathrm{~N} / \mathrm{A}$ & $\mathrm{N} / \mathrm{A}$ & $\mathrm{N} / \mathrm{A}$ & $\mathrm{N} / \mathrm{A}$ \\
\hline ERROR-CODE & $\mathrm{N} / \mathrm{A}$ & $\mathrm{N} / \mathrm{A}$ & M & $\mathrm{N} / \mathrm{A}$ & $\mathrm{N} / \mathrm{A}$ & M \\
\hline UNKNOWN-ATTRIBUTES & $\mathrm{N} / \mathrm{A}$ & $\mathrm{N} / \mathrm{A}$ & $\mathrm{C}$ & $\mathrm{N} / \mathrm{A}$ & $\mathrm{N} / \mathrm{A}$ & $\mathrm{C}$ \\
\hline REFLECTED-FROM & $\mathrm{N} / \mathrm{A}$ & $\mathrm{C}$ & $\mathrm{N} / \mathrm{A}$ & $\mathrm{N} / \mathrm{A}$ & $\mathrm{N} / \mathrm{A}$ & $\mathrm{N} / \mathrm{A}$ \\
\hline
\end{tabular}

Table 2: Summary of Attributes

The length refers to the length of the value element, expressed as an unsigned integral number of bytes.

\subsubsection{MAPPED-ADDRESS}

The MAPPED-ADDRESS attribute indicates the mapped IP address and port. It consists of an eight bit address family, and a sixteen bit port, followed by a fixed length value representing the IP address.

$\begin{array}{lllllll}0 & 1 & 2 & 3\end{array}$

$\begin{array}{llllllllllllllllllllllllllllllll}0 & 1 & 2 & 3 & 4 & 5 & 6 & 7 & 8 & 9 & 0 & 1 & 2 & 3 & 4 & 5 & 6 & 7 & 8 & 9 & 0 & 1 & 2 & 3 & 4 & 5 & 6 & 7 & 8 & 9 & 0 & 1\end{array}$ +-+-+-+-+-+-+-+-+-+-+-+-+-+-+-+-+-+-+-+-+-+-+-+-+-+-+-+-+-+-+-+-+ $|\mathrm{x} \times \mathrm{x} \times \mathrm{x} \times \mathrm{x}|$ Family $\quad$ Port +-+-+-+-+-+-+-+-+-+-+-+-+-+-+-+-+-+-+-+-+-+-+-+-+-+-+- +- +- +- +- +- + | Address

The port is a network byte ordered representation of the mapped port. The address family is always 0x01, corresponding to IPv4. The first 8 bits of the MAPPED-ADDRESS are ignored, for the purposes of aligning parameters on natural boundaries. The IPv4 address is 32 bits.

\section{2 .2 RESPONSE-ADDRESS}

The RESPONSE-ADDRESS attribute indicates where the response to a Binding Request should be sent. Its syntax is identical to MAPPEDADDRESS . 


\subsubsection{CHANGED-ADDRESS}

The CHANGED-ADDRESS attribute indicates the IP address and port where responses would have been sent from if the "change IP" and "change port" flags had been set in the CHANGE-REQUEST attribute of the Binding Request. The attribute is always present in a Binding Response, independent of the value of the flags. Its syntax is identical to MAPPED-ADDRESS .

\subsubsection{CHANGE-REQUEST}

The CHANGE-REQUEST attribute is used by the client to request that the server use a different address and/or port when sending the response. The attribute is 32 bits long, although only two bits (A and B) are used:

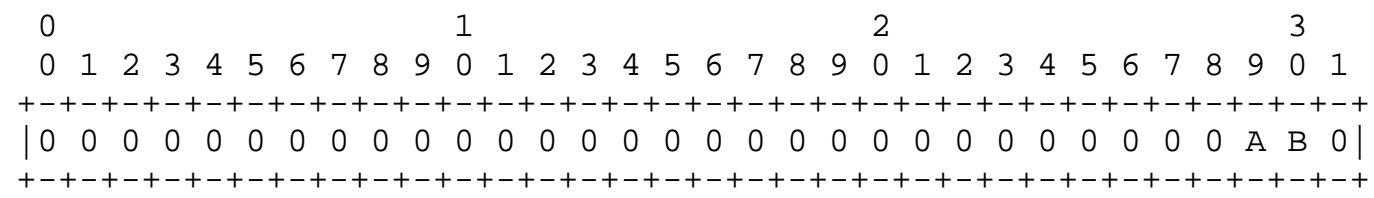

The meaning of the flags is:

A: This is the "change IP" flag. If true, it requests the server to send the Binding Response with a different IP address than the one the Binding Request was received on.

B: This is the "change port" flag. If true, it requests the server to send the Binding Response with a different port than the one the Binding Request was received on.

\subsubsection{SOURCE-ADDRESS}

The SOURCE-ADDRESS attribute is present in Binding Responses. It indicates the source IP address and port that the server is sending the response from. Its syntax is identical to that of MAPPEDADDRESS .

\subsubsection{USERNAME}

The USERNAME attribute is used for message integrity. It serves as a means to identify the shared secret used in the message integrity check. The USERNAME is always present in a shared Secret Response, along with the PASSWORD. It is optionally present in a Binding Request when message integrity is used. 
The value of USERNAME is a variable length opaque value. Its length MUST be a multiple of 4 (measured in bytes) in order to guarantee alignment of attributes on word boundaries.

\section{2 .7 PASSWORD}

The PASSWORD attribute is used in Shared Secret Responses. It is always present in a shared Secret Response, along with the USERNAME.

The value of PASSWORD is a variable length value that is to be used as a shared secret. Its length MUST be a multiple of 4 (measured in bytes) in order to guarantee alignment of attributes on word boundaries.

\subsubsection{MESSAGE-INTEGRITY}

The MESSAGE-INTEGRITY attribute contains an HMAC-SHA1 [13] of the STUN message. It can be present in Binding Requests or Binding Responses. Since it uses the SHA1 hash, the HMAC will be 20 bytes. The text used as input to HMAC is the STUN message, including the header, up to and including the attribute preceding the MESSAGEINTEGRITY attribute. That text is then padded with zeroes so as to be a multiple of 64 bytes. As a result, the MESSAGE-INTEGRITY attribute MUST be the last attribute in any STUN message. The key used as input to HMAC depends on the context.

\section{2 .9 ERROR-CODE}

The ERROR-CODE attribute is present in the Binding Error Response and Shared Secret Error Response. It is a numeric value in the range of 100 to 699 plus a textual reason phrase encoded in UTF-8, and is consistent in its code assignments and semantics with SIP [10] and HTTP [15]. The reason phrase is meant for user consumption, and can be anything appropriate for the response code. The lengths of the reason phrases MUST be a multiple of 4 (measured in bytes). This can be accomplished by added spaces to the end of the text, if necessary. Recommended reason phrases for the defined response codes are presented below.

To facilitate processing, the class of the error code (the hundreds digit) is encoded separately from the rest of the code. 


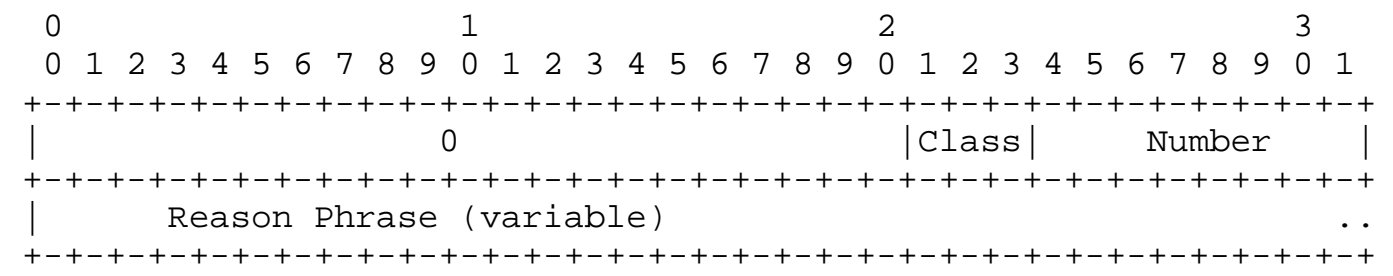

The class represents the hundreds digit of the response code. The value MUST be between 1 and 6 . The number represents the response code modulo 100, and its value MUST be between 0 and 99 .

The following response codes, along with their recommended reason phrases (in brackets) are defined at this time:

400 (Bad Request): The request was malformed. The client should not retry the request without modification from the previous attempt.

401 (Unauthorized): The Binding Request did not contain a MESSAGEINTEGRITY attribute.

420 (Unknown Attribute): The server did not understand a mandatory attribute in the request.

430 (Stale Credentials): The Binding Request did contain a MESSAGEINTEGRITY attribute, but it used a shared secret that has expired. The client should obtain a new shared secret and try again.

431 (Integrity Check Failure): The Binding Request contained a MESSAGE-INTEGRITY attribute, but the HMAC failed verification. This could be a sign of a potential attack, or client implementation error.

432 (Missing Username): The Binding Request contained a MESSAGEINTEGRITY attribute, but not a USERNAME attribute. Both must be present for integrity checks.

433 (Use TLS): The Shared Secret request has to be sent over TLS, but was not received over TLS.

500 (Server Error): The server has suffered a temporary error. The client should try again.

600 (Global Failure:) The server is refusing to fulfill the request. The client should not retry. 


\subsubsection{UNKNOWN-ATTRIBUTES}

The UNKNOWN-ATTRIBUTES attribute is present only in a Binding Error Response or Shared Secret Error Response when the response code in the ERROR-CODE attribute is 420 .

The attribute contains a list of 16 bit values, each of which represents an attribute type that was not understood by the server. If the number of unknown attributes is an odd number, one of the attributes MUST be repeated in the list, so that the total length of the list is a multiple of 4 bytes.

0 $\begin{array}{lllllllllllllllllllllllllllllllll}0 & 1 & 2 & 3 & 4 & 5 & 6 & 7 & 8 & 9 & 0 & 1 & 2 & 3 & 4 & 5 & 6 & 7 & 8 & 9 & 0 & 1 & 2 & 3 & 4 & 5 & 6 & 7 & 8 & 9 & 0 & 1\end{array}$ Attribute 1 Type | Atribute 2 Type
+-+-+-+-+-+-+-+-+-+-+-+-+-+-+-+-+-+-+-+-+-+-+-+-+-+-+-+-+-+-+-+-+
Attribute 3 Type $\quad$ Attribute 4 Type...
+-+-+-+-+-+-+-+-+-+-+-+-+-+-+-+-+-+-+-+-+-+-+-+-+-+-+-+-+-+-+-+-+

\subsubsection{REFLECTED-FROM}

The REFLECTED-FROM attribute is present only in Binding Responses, when the Binding Request contained a RESPONSE-ADDRESS attribute. The attribute contains the identity (in terms of IP address) of the source where the request came from. Its purpose is to provide traceability, so that a STUN server cannot be used as a reflector for denial-of-service attacks.

Its syntax is identical to the MAPPED-ADDRESS attribute.

12. Security Considerations

\subsection{Attacks on STUN}

Generally speaking, attacks on STUN can be classified into denial of service attacks and eavesdropping attacks. Denial of service attacks can be launched against a STUN server itself, or against other elements using the STUN protocol.

STUN servers create state through the Shared Secret Request mechanism. To prevent being swamped with traffic, a STUN server SHOULD limit the number of simultaneous TLS connections it will hold open by dropping an existing connection when a new connection request arrives (based on an Least Recently Used (LRU) policy, for example). Similarly, it SHOULD limit the number of shared secrets it will store, in the event that the server is storing the shared secrets. 
The attacks of greater interest are those in which the STUN server and client are used to launch Dos attacks against other entities, including the client itself.

Many of the attacks require the attacker to generate a response to a legitimate STUN request, in order to provide the client with a faked MAPPED-ADDRESS. The attacks that can be launched using such a technique include:

12.1.1 Attack I: DDOS Against a Target

In this case, the attacker provides a large number of clients with the same faked MAPPED-ADDRESS that points to the intended target. This will trick all the STUN clients into thinking that their addresses are equal to that of the target. The clients then hand out that address in order to receive traffic on it (for example, in SIP or H.323 messages). However, all of that traffic becomes focused at the intended target. The attack can provide substantial amplification, especially when used with clients that are using STUN to enable multimedia applications.

\subsubsection{Attack II: Silencing a Client}

In this attack, the attacker seeks to deny a client access to services enabled by STUN (for example, a client using STUN to enable SIP-based multimedia traffic). To do that, the attacker provides that client with a faked MAPPED-ADDRESS. The MAPPED-ADDRESS it provides is an IP address that routes to nowhere. As a result, the client won't receive any of the packets it expects to receive when it hands out the MAPPED-ADDRESS.

This exploitation is not very interesting for the attacker. It impacts a single client, which is frequently not the desired target. Moreover, any attacker that can mount the attack could also deny service to the client by other means, such as preventing the client from receiving any response from the STUN server, or even a DHCP server.

12.1.3 Attack III: Assuming the Identity of a Client

This attack is similar to attack II. However, the faked MAPPEDADDRESS points to the attacker themself. This allows the attacker to receive traffic which was destined for the client. 


\subsubsection{Attack IV: Eavesdropping}

In this attack, the attacker forces the client to use a MAPPEDADDRESS that routes to itself. It then forwards any packets it receives to the client. This attack would allow the attacker to observe all packets sent to the client. However, in order to launch the attack, the attacker must have already been able to observe packets from the client to the STUN server. In most cases (such as when the attack is launched from an access network), this means that the attacker could already observe packets sent to the client. This attack is, as a result, only useful for observing traffic by attackers on the path from the client to the STUN server, but not generally on the path of packets being routed towards the client.

\subsection{Launching the Attacks}

It is important to note that attacks of this nature (injecting responses with fake MAPPED-ADDRESSes) require that the attacker be capable of eavesdropping requests sent from the client to the server (or to act as a MITM for such attacks). This is because STUN requests contain a transaction identifier, selected by the client, which is random with 128 bits of entropy. The server echoes this value in the response, and the client ignores any responses that don't have a matching transaction ID. Therefore, in order for an attacker to provide a faked response that is accepted by the client, the attacker needs to know what the transaction ID in the request was. The large amount of randomness, combined with the need to know when the client sends a request, precludes attacks that involve guessing the transaction ID.

Since all of the above attacks rely on this one primitive - injecting a response with a faked MAPPED-ADDRESS - preventing the attacks is accomplished by preventing this one operation. To prevent it, we need to consider the various ways in which it can be accomplished. There are several:

\subsubsection{Approach I: Compromise a Legitimate STUN Server}

In this attack, the attacker compromises a legitimate STUN server through a virus or Trojan horse. Presumably, this would allow the attacker to take over the STUN server, and control the types of responses it generates.

Compromise of a STUN server can also lead to discovery of open ports. Knowledge of an open port creates an opportunity for Dos attacks on those ports (or DDoS attacks if the traversed NAT is a full cone NAT). Discovering open ports is already fairly trivial using port probing, so this does not represent a major threat. 


\subsubsection{Approach II: DNS Attacks}

STUN servers are discovered using DNS SRV records. If an attacker can compromise the DNS, it can inject fake records which map a domain name to the IP address of a STUN server run by the attacker. This will allow it to inject fake responses to launch any of the attacks above.

\subsubsection{Approach III: Rogue Router or NAT}

Rather than compromise the STUN server, an attacker can cause a STUN server to generate responses with the wrong MAPPED-ADDRESS by compromising a router or NAT on the path from the client to the STUN server. When the STUN request passes through the rogue router or NAT, it rewrites the source address of the packet to be that of the desired MAPPED-ADDRESS. This address cannot be arbitrary. If the attacker is on the public Internet (that is, there are no NATs between it and the STUN server), and the attacker doesn't modify the STUN request, the address has to have the property that packets sent from the STUN server to that address would route through the compromised router. This is because the STUN server will send the responses back to the source address of the request. With a modified source address, the only way they can reach the client is if the compromised router directs them there. If the attacker is on the public Internet, but they can modify the STUN request, they can insert a RESPONSE-ADDRESS attribute into the request, containing the actual source address of the STUN request. This will cause the server to send the response to the client, independent of the source address the STUN server sees. This gives the attacker the ability to forge an arbitrary source address when it forwards the STUN request.

If the attacker is on a private network (that is, there are NATs between it and the STUN server), the attacker will not be able to force the server to generate arbitrary MAPPED-ADRESSes in responses. They will only be able force the STUN server to generate MAPPEDADDRESSes which route to the private network. This is because the NAT between the attacker and the STUN server will rewrite the source address of the STUN request, mapping it to a public address that routes to the private network. Because of this, the attacker can only force the server to generate faked mapped addresses that route to the private network. Unfortunately, it is possible that a low quality NAT would be willing to map an allocated public address to another public address (as opposed to an internal private address), in which case the attacker could forge the source address in a STUN request to be an arbitrary public address. This kind of behavior from NATs does appear to be rare. 


\subsubsection{Approach IV: MITM}

As an alternative to approach III, if the attacker can place an element on the path from the client to the server, the element can act as a man-in-the-middle. In that case, it can intercept a STUN request, and generate a STUN response directly with any desired value of the MAPPED-ADDRESS field. Alternatively, it can forward the STUN request to the server (after potential modification), receive the response, and forward it to the client. When forwarding the request and response, this attack is subject to the same limitations on the MAPPED-ADDRESS described in Section 12.2.3.

\subsubsection{Approach V: Response Injection Plus DoS}

In this approach, the attacker does not need to be a MITM (as in approaches III and IV). Rather, it only needs to be able to eavesdrop onto a network segment that carries STUN requests. This is easily done in multiple access networks such as ethernet or unprotected 802.11. To inject the fake response, the attacker listens on the network for a STUN request. When it sees one, it simultaneously launches a Dos attack on the STUN server, and generates its own STUN response with the desired MAPPED-ADDRESS value. The STUN response generated by the attacker will reach the client, and the Dos attack against the server is aimed at preventing the legitimate response from the server from reaching the client. Arguably, the attacker can do without the Dos attack on the server, so long as the faked response beats the real response back to the client, and the client uses the first response, and ignores the second (even though it's different).

\subsubsection{Approach VI: Duplication}

This approach is similar to approach V. The attacker listens on the network for a STUN request. When it sees it, it generates its own STUN request towards the server. This STUN request is identical to the one it saw, but with a spoofed source IP address. The spoofed address is equal to the one that the attacker desires to have placed in the MAPPED-ADDRESS of the STUN response. In fact, the attacker generates a flood of such packets. The STUN server will receive the one original request, plus a flood of duplicate fake ones. It generates responses to all of them. If the flood is sufficiently large for the responses to congest routers or some other equipment, there is a reasonable probability that the one real response is lost (along with many of the faked ones), but the net result is that only the faked responses are received by the STUN client. These responses are all identical and all contain the MAPPED-ADDRESS that the attacker wanted the client to use. 
The flood of duplicate packets is not needed (that is, only one faked request is sent), so long as the faked response beats the real response back to the client, and the client uses the first response, and ignores the second (even though it's different).

Note that, in this approach, launching a Dos attack against the STUN server or the IP network, to prevent the valid response from being sent or received, is problematic. The attacker needs the STUN server to be available to handle its own request. Due to the periodic retransmissions of the request from the client, this leaves a very tiny window of opportunity. The attacker must start the Dos attack immediately after the actual request from the client, causing the correct response to be discarded, and then cease the Dos attack in order to send its own request, all before the next retransmission from the client. Due to the close spacing of the retransmits (100ms to a few seconds), this is very difficult to do.

Besides Dos attacks, there may be other ways to prevent the actual request from the client from reaching the server. Layer 2 manipulations, for example, might be able to accomplish it.

Fortunately, Approach IV is subject to the same limitations documented in Section 12.2.3, which limit the range of MAPPEDADDRESSes the attacker can cause the STUN server to generate.

\subsection{Countermeasures}

STUN provides mechanisms to counter the approaches described above, and additional, non-STUN techniques can be used as well.

First off, it is RECOMMENDED that networks with STUN clients implement ingress source filtering (RFC 2827 [7]). This is particularly important for the NATs themselves. As Section 12.2.3 explains, NATs which do not perform this check can be used as "reflectors" in DDos attacks. Most NATs do perform this check as a default mode of operation. We strongly advise people that purchase NATs to ensure that this capability is present and enabled.

Secondly, it is RECOMMENDED that STUN servers be run on hosts dedicated to STUN, with all UDP and TCP ports disabled except for the STUN ports. This is to prevent viruses and Trojan horses from infecting STUN servers, in order to prevent their compromise. This helps mitigate Approach I (Section 12.2.1).

Thirdly, to prevent the DNS attack of Section 12.2.2, Section 9.2 recommends that the client verify the credentials provided by the server with the name used in the DNS lookup. 
Finally, all of the attacks above rely on the client taking the mapped address it learned from STUN, and using it in application layer protocols. If encryption and message integrity are provided within those protocols, the eavesdropping and identity assumption attacks can be prevented. As such, applications that make use of STUN addresses in application protocols sHoULD use integrity and encryption, even if a SHOULD level strength is not specified for that protocol. For example, multimedia applications using STUN addresses to receive RTP traffic would use secure RTP [16].

The above three techniques are non-STUN mechanisms. STUN itself provides several countermeasures.

Approaches IV (Section 12.2.4), when generating the response locally, and $V$ (Section 12.2.5) require an attacker to generate a faked response. This attack is prevented using the message integrity mechanism provided in STUN, described in section 8.1.

Approaches III (Section 12.2.3) IV (Section 12.2.4), when using the relaying technique, and VI (12.2.6), however, are not preventable through server signatures. Both approaches are most potent when the attacker can modify the request, inserting a RESPONSE-ADDRESS that routes to the client. Fortunately, such modifications are preventable using the message integrity techniques described in Section 9.3. However, these three approaches are still functional when the attacker modifies nothing but the source address of the STUN request. Sadly, this is the one thing that cannot be protected through cryptographic means, as this is the change that sTUN itself is seeking to detect and report. It is therefore an inherent weakness in NAT, and not fixable in STUN. To help mitigate these attacks, Section 9.4 provides several heuristics for the client to follow. The client looks for inconsistent or extra responses, both of which are signs of the attacks described above. However, these heuristics are just that - heuristics, and cannot be guaranteed to prevent attacks. The heuristics appear to prevent the attacks as we know how to launch them today. Implementors should stay posted for information on new heuristics that might be required in the future. Such information will be distributed on the IETF MIDCOM mailing list, midcomeietf.org.

\section{4 Residual Threats}

None of the countermeasures listed above can prevent the attacks described in section 12.2.3 if the attacker is in the appropriate network paths. Specifically, consider the case in which the attacker wishes to convince client $\mathrm{C}$ that it has address $\mathrm{V}$. The attacker needs to have a network element on the path between $A$ and the server (in order to modify the request) and on the path between the server 
and $V$ so that it can forward the response to $C$. Furthermore, if there is a NAT between the attacker and the server, $V$ must also be behind the same NAT. In such a situation, the attacker can either gain access to all the application-layer traffic or mount the DDOS attack described in section 12.1.1. Note that any host which exists in the correct topological relationship can be DDOSed. It need not be using STUN.

\section{IANA Considerations}

STUN cannot be extended. Changes to the protocol are made through a standards track revision of this specification. As a result, no IANA registries are needed. Any future extensions will establish any needed registries.

\section{IAB Considerations}

The IAB has studied the problem of "Unilateral Self Address Fixing", which is the general process by which a client attempts to determine its address in another realm on the other side of a NAT through a collaborative protocol reflection mechanism (RFC 3424 [17]). STUN is an example of a protocol that performs this type of function. The IAB has mandated that any protocols developed for this purpose document a specific set of considerations. This section meets those requirements.

\subsection{Problem Definition}

From RFC 3424 [17], any UNSAF proposal must provide:

Precise definition of a specific, limited-scope problem that is to be solved with the UNSAF proposal. A short term fix should not be generalized to solve other problems; this is why "short term fixes usually aren't".

The specific problems being solved by STUN are:

- Provide a means for a client to detect the presence of one or more NATs between it and a server run by a service provider on the public Internet. The purpose of such detection is to determine additional steps that might be necessary in order to receive service from that particular provider.

- Provide a means for a client to detect the presence of one or more NATs between it and another client, where the second client is reachable from the first, but it is not known whether the second client resides on the public Internet. 
- Provide a means for a client to obtain an address on the public Internet from a non-symmetric NAT, for the express purpose of receiving incoming UDP traffic from another host, targeted to that address.

STUN does not address TCP, either incoming or outgoing, and does not address outgoing UDP communications.

\subsection{Exit Strategy}

From [17], any UNSAF proposal must provide:

Description of an exit strategy/transition plan. The better short term fixes are the ones that will naturally see less and less use as the appropriate technology is deployed.

STUN comes with its own built in exit strategy. This strategy is the detection operation that is performed as a precursor to the actual UNSAF address-fixing operation. This discovery operation, documented in Section 10.1, attempts to discover the existence of, and type of, any NATS between the client and the service provider network. Whilst the detection of the specific type of NAT may be brittle, the discovery of the existence of NAT is itself quite robust. As NATs are phased out through the deployment of IPv6, the discovery operation will return immediately with the result that there is no NAT, and no further operations are required. Indeed, the discovery operation itself can be used to help motivate deployment of IPv6; if a user detects a NAT between themselves and the public Internet, they can call up their access provider and complain about it.

STUN can also help facilitate the introduction of midcom. As midcom-capable NATs are deployed, applications will, instead of using STUN (which also resides at the application layer), first allocate an address binding using midcom. However, it is a well-known limitation of midcom that it only works when the agent knows the middleboxes through which its traffic will flow. Once bindings have been allocated from those middleboxes, a STUN detection procedure can validate that there are no additional middleboxes on the path from the public Internet to the client. If this is the case, the application can continue operation using the address bindings allocated from midcom. If it is not the case, STUN provides a mechanism for self-address fixing through the remaining midcomunaware middleboxes. Thus, STUN provides a way to help transition to full midcom-aware networks. 


\subsection{Brittleness Introduced by STUN}

From [17], any UNSAF proposal must provide:

Discussion of specific issues that may render systems more "brittle". For example, approaches that involve using data at multiple network layers create more dependencies, increase debugging challenges, and make it harder to transition.

STUN introduces brittleness into the system in several ways:

- The discovery process assumes a certain classification of devices based on their treatment of UDP. There could be other types of NATs that are deployed that would not fit into one of these molds. Therefore, future NATs may not be properly detected by STUN. STUN clients (but not servers) would need to change to accommodate that.

- The binding acquisition usage of STUN does not work for all NAT types. It will work for any application for full cone NATs only. For restricted cone and port restricted cone NAT, it will work for some applications depending on the application. Application specific processing will generally be needed. For symmetric NATs, the binding acquisition will not yield a usable address. The tight dependency on the specific type of NAT makes the protocol brittle.

- STUN assumes that the server exists on the public Internet. If the server is located in another private address realm, the user may or may not be able to use its discovered address to communicate with other users. There is no way to detect such a condition.

- The bindings allocated from the NAT need to be continuously refreshed. Since the timeouts for these bindings is very implementation specific, the refresh interval cannot easily be determined. When the binding is not being actively used to receive traffic, but to wait for an incoming message, the binding refresh will needlessly consume network bandwidth.

- The use of the STUN server as an additional network element introduces another point of potential security attack. These attacks are largely prevented by the security measures provided by STUN, but not entirely. 
- The use of the STUN server as an additional network element introduces another point of failure. If the client cannot locate a STUN server, or if the server should be unavailable due to failure, the application cannot function.

- The use of STUN to discover address bindings will result in an increase in latency for applications. For example, a Voice over IP application will see an increase of call setup delays equal to at least one RTT to the STUN server.

- The discovery of binding lifetimes is prone to error. It assumes that the same lifetime will exist for all bindings. This may not be true if the NAT uses dynamic binding lifetimes to handle overload, or if the NAT itself reboots during the discovery process.

- STUN imposes some restrictions on the network topologies for proper operation. If client A obtains an address from STUN server $X$, and sends it to client $B, B$ may not be able to send to A using that IP address. The address will not work if any of the following is true:

- The STUN server is not in an address realm that is a common ancestor (topologically) of both clients A and B. For example, consider client $A$ and $B$, both of which have residential NAT devices. Both devices connect them to their cable operators, but both clients have different providers. Each provider has a NAT in front of their entire network, connecting it to the public Internet. If the STUN server used by $A$ is in $A^{\prime} S$ cable operator's network, an address obtained by it will not be usable by B. The STUN server must be in the network which is a common ancestor to both - in this case, the public Internet.

- The STUN server is in an address realm that is a common ancestor to both clients, but both clients are behind the same NAT connecting to that address realm. For example, if the two clients in the previous example had the same cable operator, that cable operator had a single NAT connecting their network to the public Internet, and the STUN server was on the public Internet, the address obtained by A would not be usable by B. That is because some NATs will not accept an internal packet sent to a public IP address which is mapped back to an internal address. To deal with this, additional protocol mechanisms or configuration parameters need to be introduced which detect this case. 
- Most significantly, STUN introduces potential security threats which cannot be eliminated. This specification describes heuristics that can be used to mitigate the problem, but it is provably unsolvable given what STUN is trying to accomplish. These security problems are described fully in section 12 .

14.4 Requirements for a Long Term Solution

From [17], any UNSAF proposal must provide:

Identify requirements for longer term, sound technical solutions -- contribute to the process of finding the right longer term solution.

Our experience with STUN has led to the following requirements for a long term solution to the NAT problem:

Requests for bindings and control of other resources in a NAT need to be explicit. Much of the brittleness in STUN derives from its guessing at the parameters of the NAT, rather than telling the NAT what parameters to use.

Control needs to be "in-band". There are far too many scenarios in which the client will not know about the location of middleboxes ahead of time. Instead, control of such boxes needs to occur in-band, traveling along the same path as the data will itself travel. This guarantees that the right set of middleboxes are controlled. This is only true for first-party controls; third-party controls are best handled using the midcom framework.

Control needs to be limited. Users will need to communicate through NATs which are outside of their administrative control. In order for providers to be willing to deploy NATs which can be controlled by users in different domains, the scope of such controls needs to be extremely limited - typically, allocating a binding to reach the address where the control packets are coming from.

Simplicity is paramount. The control protocol will need to be implement in very simple clients. The servers will need to support extremely high loads. The protocol will need to be extremely robust, being the precursor to a host of application protocols. As such, simplicity is key. 


\subsection{Issues with Existing NAPT Boxes}

From [17], any UNSAF proposal must provide:

Discussion of the impact of the noted practical issues with existing, deployed NA[P]Ts and experience reports.

Several of the practical issues with STUN involve future proofing breaking the protocol when new NAT types get deployed. Fortunately, this is not an issue at the current time, since most of the deployed NATs are of the types assumed by STUN. The primary usage STUN has found is in the area of VoIP, to facilitate allocation of addresses for receiving RTP [12] traffic. In that application, the periodic keepalives are provided by the RTP traffic itself. However, several practical problems arise for RTP. First, RTP assumes that RTCP traffic is on a port one higher than the RTP traffic. This pairing property cannot be guaranteed through NATs that are not directly controllable. As a result, RTCP traffic may not be properly received. Protocol extensions to SDP have been proposed which mitigate this by allowing the client to signal a different port for RTCP [18]. However, there will be interoperability problems for some time.

For VoIP, silence suppression can cause a gap in the transmission of RTP packets. This could result in the loss of a binding in the middle of a call, if that silence period exceeds the binding timeout. This can be mitigated by sending occasional silence packets to keep the binding alive. However, the result is additional brittleness; proper operation depends on the silence suppression algorithm in use, the usage of a comfort noise codec, the duration of the silence period, and the binding lifetime in the NAT.

\subsection{In Closing}

The problems with STUN are not design flaws in STUN. The problems in STUN have to do with the lack of standardized behaviors and controls in NATs. The result of this lack of standardization has been a proliferation of devices whose behavior is highly unpredictable, extremely variable, and uncontrollable. STUN does the best it can in such a hostile environment. Ultimately, the solution is to make the environment less hostile, and to introduce controls and standardized behaviors into NAT. However, until such time as that happens, STUN provides a good short term solution given the terrible conditions under which it is forced to operate. 


\section{Acknowledgments}

The authors would like to thank Cedric Aoun, Pete Cordell, Cullen Jennings, Bob Penfield and Chris sullivan for their comments, and Baruch Sterman and Alan Hawrylyshen for initial implementations. Thanks for Leslie Daigle, Allison Mankin, Eric Rescorla, and Henning Schulzrinne for IESG and IAB input on this work.

16. Normative References

[1] Bradner, S., "Key words for use in RFCs to indicate requirement levels", BCP 14, RFC 2119, March 1997.

[2] Dierks, T. and C. Allen, "The TLS protocol Version 1.0", RFC 2246, January 1999.

[3] Gulbrandsen, A., Vixie, P. and L. Esibov, "A DNS RR for specifying the location of services (DNS SRV)", RFC 2782, February 2000 .

[4] Chown, P., "Advanced Encryption Standard (AES) Ciphersuites for Transport Layer Security (TLS)", RFC 3268, June 2002.

[5] Rescorla, E., "HTTP over TLS", RFC 2818, May 2000.

[6] Postel, J., "Internet Protocol", STD 5, RFC 791, September 1981.

[7] Ferguson, P. and D. Senie, "Network Ingress Filtering: Defeating Denial of Service Attacks which employ IP Source Address Spoofing", BCP 38, RFC 2827, May 2000.

17. Informative References

[8] Senie, D., "Network Address Translator (NAT)-Friendly Application Design Guidelines", RFC 3235, January 2002.

[9] Srisuresh, P., Kuthan, J., Rosenberg, J., Molitor, A. and A. Rayhan, "Middlebox Communication Architecture and Framework", RFC 3303, August 2002 .

[10] Rosenberg, J., Schulzrinne, H., Camarillo, G., Johnston, A., Peterson, J., Sparks, R., Handley, M. and E. Schooler, "SIP: Session Initiation Protocol", RFC 3261, June 2002.

[11] Holdrege, M. and P. Srisuresh, "Protocol Complications with the IP Network Address Translator", RFC 3027, January 2001. 
[12] Schulzrinne, H., Casner, S., Frederick, R. and V. Jacobson, "RTP: A Transport Protocol for Real-Time Applications", RFC 1889, January 1996.

[13] Krawczyk, H., Bellare, M. and R. Canetti, "HMAC: Keyed-Hashing for Message Authentication", RFC 2104, February 1997.

[14] Kohl, J. and C. Neuman, "The kerberos Network Authentication Service (V5)", RFC 1510, September 1993.

[15] Fielding, R., Gettys, J., Mogul, J., Frystyk, H., Masinter, L., Leach, P. and T. Berners-Lee, "Hypertext Transfer Protocol -HTTP/1.1", RFC 2616, June 1999.

[16] Baugher M., et al., "The secure real-time transport protocol", Work in Progress.

[17] Daigle, L., Editor, "IAB Considerations for UNilateral SelfAddress Fixing (UNSAF) Across Network Address Translation", RFC 3424 , November 2002 .

[18] Huitema, C., "RTCP attribute in SDP", Work in Progress. 
18. Authors' Addresses

Jonathan Rosenberg

dynamicsoft

72 Eagle Rock Avenue

First Floor

East Hanover, NJ 07936

EMail: jdrosen@dynamicsoft.com

Joel Weinberger

dynamicsoft

72 Eagle Rock Avenue

First Floor

East Hanover, NJ 07936

EMail: jweinberger@dynamicsoft.com

Christian Huitema

Microsoft Corporation

One Microsoft Way

Redmond, WA 98052-6399

EMail: huitema@microsoft.com

Rohan Mahy

Cisco Systems

101 Cooper St

Santa Cruz, CA 95060

EMail: rohan@cisco.com 
19. Full Copyright statement

Copyright (C) The Internet Society (2003). All Rights Reserved.

This document and translations of it may be copied and furnished to others, and derivative works that comment on or otherwise explain it or assist in its implementation may be prepared, copied, published and distributed, in whole or in part, without restriction of any kind, provided that the above copyright notice and this paragraph are included on all such copies and derivative works. However, this document itself may not be modified in any way, such as by removing the copyright notice or references to the Internet society or other Internet organizations, except as needed for the purpose of developing Internet standards in which case the procedures for copyrights defined in the Internet Standards process must be followed, or as required to translate it into languages other than English.

The limited permissions granted above are perpetual and will not be revoked by the Internet society or its successors or assigns.

This document and the information contained herein is provided on an "AS IS" basis and THE INTERNET SOCIETY AND THE INTERNET ENGINEERING TASK FORCE DISCLAIMS ALL WARRANTIES, EXPRESS OR IMPLIED, INCLUDING BUT NOT LIMITED TO ANY WARRANTY THAT THE USE OF THE INFORMATION HEREIN WILL NOT INFRINGE ANY RIGHTS OR ANY IMPLIED WARRANTIES OF MERCHANTABILITY OR FITNESS FOR A PARTICULAR PURPOSE.

Acknowledgement

Funding for the RFC Editor function is currently provided by the Internet society. 